\title{
Eine Folge von Eem und 4 Weichsel-Interstadialen in Oerel/Niedersachsen und ihr Vegetationsablauf
}

\author{
KarL-ERnst Behre \& Udo Lade *)
}

\begin{abstract}
Geological sections, Eemian, Weichselian interstadials, limnic sediments, peat, sand, pollen diagrams, vegetation, forests, tundra, paleoclimate

Northwest German Lowlands (Oerel), Lower Saxony

TK 25: Nr. 2520
\end{abstract}

\begin{abstract}
Kurzfassung: An dem Interstadialvorkommen von Oerel im niedersächsischen Tiefland wurden umfangreiche geologische und vegetationsgeschichtliche Untersuchungen durchgeführt. Die Lokalität liegt in einer buchtförmigen Niederung am Osthang der Eisrandlage Lamstedter Staffel (Drenthe-2-Vorstoß der Saale-Kaltzeit). Sondierungen und Bohrungen ergaben ein ausgedehntes Eem-Vorkommen und darüber bis zu 4 durch Sande getrennte Weichsel-Interstadiale, die flächig ausgebildet sind. In der Umgebung des pollenanalytisch bearbeiteten Standardprofils OE 61 verlief die Entwicklung wie folgt: An der Basis liegen wahrscheinlich elster- sowie saalezeitliche Bildungen. Das Eem beginnt mit Abschnitt IV und ist bis VI limnisch, anschließend bis EVII sowie teilweise im Abschnitt WF I des Weichselfrühglazials als Torf ausgebildet. In den verschiedenen Stadialen wurde überwiegend reiner Sand abgelagert, dem Brörupund Odderade-Interstadial gehen jedoch noch längere limnische Phasen voraus. Beide Interstadiale sind ebenso wie die jüngeren Interstadiale Oerel und Glinde als Torf ausgebildet. Die organogene Schichtenfolge im Bereich von OE 61 wird wie folgt eingeordnet bzw. neu benannt:
\end{abstract}

\section{Glinde-Interstadial \\ Oerel-Interstadial \\ Odderade-Interstadial \\ Brörup-Interstadial \\ Eem-Interglazial}

Nach den pollenanalytischen Untersuchungen ist das Eem normal ausgebildet; Brörup und Odderade stellen zwei große bewaldete Interstadiale dar. Oerel und Glinde waren waldfrei mit Strauchtundra und gehören dem frühen Weichsel-Pleniglazial an, liegen also vor den niederländischen pleniglazialen Interstadialen. Vielleicht reicht das Glinde noch in den Beginn des Moershoofd-Komplexes.

*) Anschrift der Autoren: Prof. Dr. K.-E. BeHre, Niedersächsisches Landesinstitut für Marschen- und Wurtenforschung, Viktoriastr. 26/28, D-2940 Wilhelmshaven. Dr. U. LADE, Prozeßmoorweg 13, D-2174 HechthausenBornberg.
[A Sequence of Eemian and 4 Weichselian Interstadials in Oerel/Niedersachsen (West Germany) and their Vegetation]

Abstract: Extensive investigations into geology and vegetational history of the Weichselian interstadial site of Oerel in the lowland of Niedersachsen (W. Germany) were carried out. The locality is situated in the eastern hinterland of the Lamstedter Staffel moraines (Drenthe-2-advance of the Saale-glaciation). Numerous borings showed a wide Eemian interglacial layer and on top of it up to 4 organic layers of Weichselian interstadials, separated from each other by sand. At the site of the standard profile OE 61, the development took place as follows: the basal layers are represented by glacial deposits of probably Elsterian and Saalian age. The Eemian starts with a limnic phase from zone IV to VI which is followed by peat until zone WF I of the Early Weichselian. During the stadials mainly pure sands were deposited, the Brörup- and Odderade-interstadials, however, are preceded by limnic phases. Both interstadials as well as the younger interstadials Oerel and Glinde are developed as peat layers. The organic sequence in the area of $\mathrm{OE} 61$ was correlated or new described as follows:

Glinde-interstadial
Oerel-interstadial
Odderade-interstadial
Brörup-interstadial
Eem-interglacial

The pollen diagrams show a normal development of the Eemian vegetation; Brörup and Odderade represent two great forested interstadials. Oerel and Glinde were treeless with shrub-tundra and were parts of the early pleniglacial of the Weichselian, i. e. they have to be placed before the pleniglacial interstadials of the Netherlands. The Glinde may perhaps be placed at the beginning of the Moershoofdcomplex. 


\section{Inhalt}

1. Einleitung

2. Geologie und Morphologie

2.1. Arbeitsmethodik

2.2. Geologischer Überblick

2.3. Oerel und Umgebung

3. Die Hohlform von Oerel

4. Vegetationsablauf und zeitliche Einordnung

4.1. Allgemeines zu den botanischen Untersuchungen

4.2. Das Eem-Interglazial und die Grenze Eem-Weichsel

4.3. Das Brörup-Interstadial

4.4. Das Odderade-Interstadial

4.5. Das Oerel-Interstadial

4.6. Das Glinde-Interstadial

4.7. Zur zeitlichen Einordnung der Interstadiale Oerel und Glinde nach dem Vegetationsbild

12

12

14

14

16

5. Diskussion

6. Schriftenverzeichnis

\section{Einleitung}

Unsere Kenntnisse über den Ablauf und den klimatischen Charakter der jüngsten, der Weichsel-Kaltzeit haben in den letzten Jahren erhebliche Fortschritte gemacht, aber auch neue Probleme aufgeworfen. Im norddeutsch-niederländischen Tiefland sind an verschiedenen Stellen wichtige Vorkommen von Weichsel-Interstadialen entdeckt und bearbeitet worden. Deren Ergebnisse liefern eine Grundvorstellung zur Entwicklung von Klima und Vegetation. Eine größere Bedeutung für diese Thematik erlangten inzwischen einige lang durchlaufende Profile aus dem südlichen Mitteleuropa, die große Teile der Weichsel( = Würm-) Kaltzeit mit mehreren Interstadialen erfaßten; nach entsprechend langen Profilen aus nördlicheren Gebieten wurde seit langem gesucht.

Die Lokalität Oerel, im Elbe-Weser-Dreieck bei Bremervörde gelegen, ist seit den Untersuchungen SELLEs bekannt. Er fand bereits 1940 über dem Eem zwei große Interstadiale, die er auf Pollen und Makroresté hin bearbeitete. Im Hangenden dieser Interstadiale stieß er auf einen weiteren Torf, von dem jedoch nur 2 Proben untersucht wurden. SELLE verstarb vor Abschluß dieser Arbeiten, aus seinem Nachlaß hat
SCHNEEKLOTH das vorhandene Material so weit wie möglich ergänzt und publiziert (SELLE \& SCHNEEKLOTH 1965, SCHNEEKLOTH 1966). Dabei wurden die beiden großen, bewaldeten Interstadiale zeitlich in das Brörup und Odderade eingeordnet. Wegen der so erlangten Bedeutung, besonders aber wegen des hangenden Torfes, war eine Neubearbeitung der Lokalität Oerel seit langem wünschenswert.

Neue geologische Untersuchungen in Oerel wurden 1976 von U. LADE begonnen (publ. 1980), bis 1981 zusammen mit H. HAGEDORN im Rahmen eines DFG - geförderten Forschungsprojekts fortgesetzt und bis 1985 von U. LADE abgeschlossen. Dabei wurde außer dem erwarteten dritten sogar noch ein vierter weichselinterstadialer Torf angetroffen. - Parallel zur geologischen übernahm K.-E. BEHRE die vegetationsgeschichtliche Bearbeitung, von der die Pollendiagramme hier vorgelegt werden, während über die noch laufenden botanischen Makrorestanalysen ebenso wie über die in Groningen in Arbeit befindlichen ${ }^{14} \mathrm{C}$-Datierungen später berichtet werden wird.

Bei der technischen Durchführung unserer Arbeiten erfuhren wir vielerlei Unterstützung. So wurde mit Hilfe des Niedersächsischen Landesamtes für Bodenforschung, Hannover, im Rahmen des vom BMFT geförderten Vorhabens RG 81021 „Aufsuchung und Untersuchung von Schwermineral-Anreicherungen im Gebiet zwischen Cuxhaven und Bremerhaven" eine kombinierte Spül-Schlauchkern-Bohrung bis auf 51,0 m Tiefe abgeteuft. Die Laborarbeiten erledigten Frau M. NÜSSEN und Frau E. SCHULZ, während die Zeichnungen von den Herren S. HAUPT und W. WISSELINCK, alle Wilhelmshaven, erstellt wurden. Ihnen allen gilt unser Dank.

Hilfreiche wissenschaftliche Diskussionen zum Thema wurden mit den Herren Priv.-Doz. Dr. E. GRÜGER, Göttingen, Prof. Dr. H. HAGEDORN, Würzburg, und Dr. B. MENKE, Kiel, geführt.

Schließlich bedanken wir uns bei allen Oereler Grundstückseigentümern, auf deren Gelände wir unsere Sondierungen und Bohrungen abteufen durften, wofür oftmals die Hausgärten benutzt werden mußten.

\section{Geologie und Morphologie}

\subsection{Arbeitsmethodik}

Ausgehend vom Standort der alten Brunnenbohrung (SELLE \& SCHNEEKLOTH 1965; hier: OE 14) wurden an 61 verschiedenen Stellen Bodenprofile gewonnen. Sie wurden fast alle entlang von Profillinien angeordnet (vgl. Abb. 3), mit Oerel (OE) bezeichnet und fortlaufend numeriert. Meist waren es Schlitzsondierungen, 
hinzu kamen verrohrte Bohrungen zur Bergung von ungestörtem Probenmaterial. Diese wurden meist in Form von kombinierten Spül-Kern-Bohrungen durchgeführt (Probennahme mit Rammkernsonde, Innen$\varnothing: 22 \mathrm{~mm}$ ). Sämtlichte Bohr- und Sondierpunkte wurden anschließend eingemessen und nivelliert. Zusammen mit 4 Baugrundsondierungen für das Gebäude der Volksbank (direkt nördlich OE 54 und 58; vgl. Abb. 3) standen 65 Profile für die Auswertung zur Verfügung.

Das wichtigste Profil ist die kombinierte SpülSchlauchkern-Bohrung OE 61 ( = WEM 39 des oben genannten Schwermineral-Forschungsprogrammes). Sie wurde bis $21,5 \mathrm{~m}$ unter Geländeoberfläche gekernt und anschließend bis $51,0 \mathrm{~m}$ gespült. Ihre Kerne $(\varnothing: 100 \mathrm{~mm})$ enthielten organogene Ablagerungen von 4 Weichsel-Interstadialen und der EemWarmzeit. Die organogene Strecke von OE 61 wurde deshalb als Standardprofil ausgewählt und nach allen Richtungen hin intensiv bearbeitet. Aus den tieferen glaziären Schichten stand ausreichendes Material für 3 Feinkiesanalysen zur Verfügung (vgl. Abb. 10).

Zur Ergänzung und um die in ihren Tiefenlagen z. T. stark schwankenden gleichalten organogenen Schichten korrelieren zu können, wurden an Mudden und Torfen folgender Sondierungen und Bohrungen pollenanalytische Übersichtsdatierungen vorgenommen: OE $9,11,16,22,23,25,45,46,48$ und 60 .

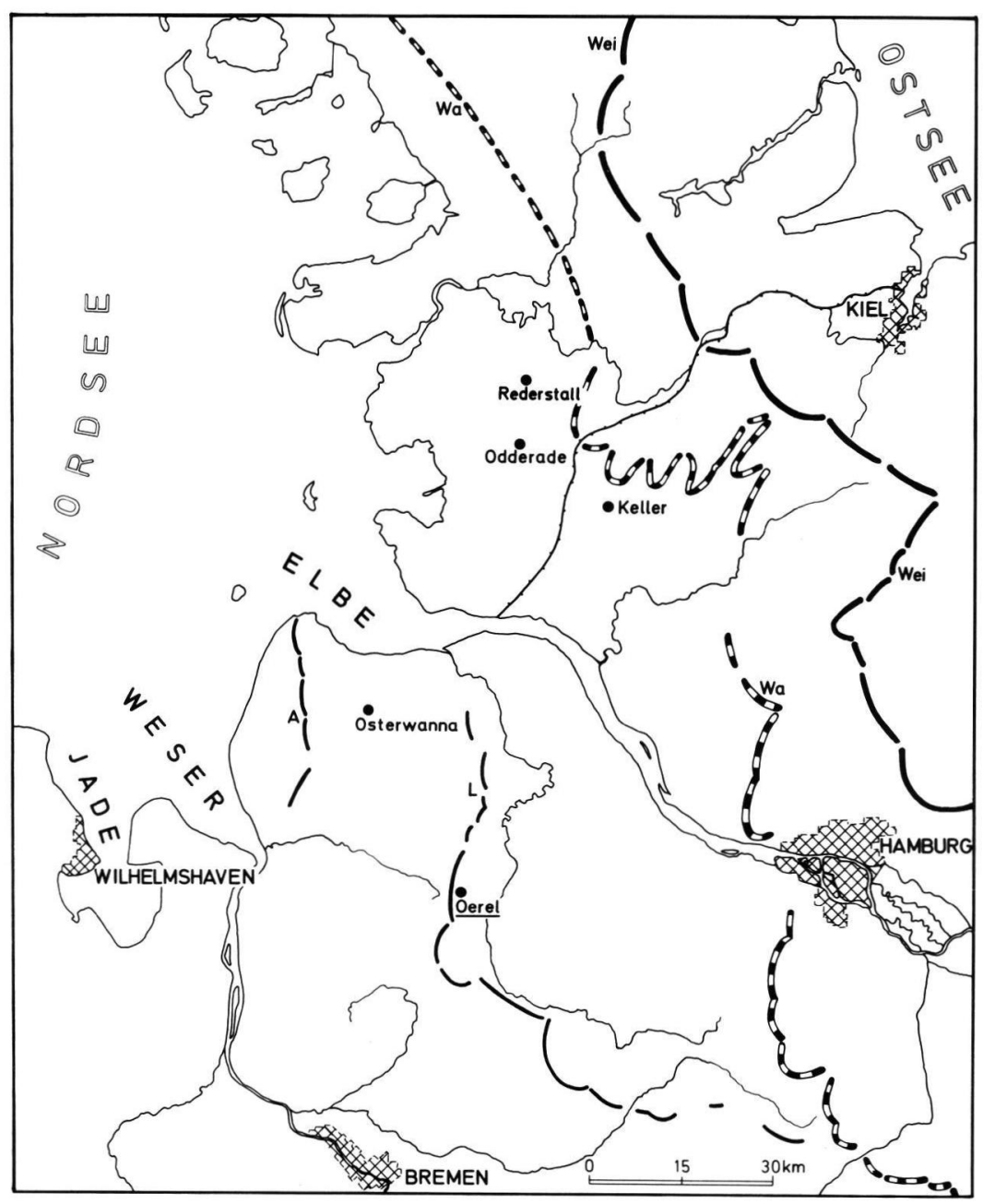

Abb. 1: Geologische Übersicht mit Eisrandlagen und wichtigen im Text genannten Fundplätzen.

Wei = Weichsel-Endmoränen, Wa $=$ Warthe-Endmoränen, L = Lamstedter Staffel, A = Altenwalder Staffel (nach LIEDTKE 1981, verändert). 


\subsection{Geologischer Überblick}

Der Ort Oerel liegt im nördlichen Niedersachsen im Elbe-Weser-Dreieck. Dieses Gebiet gliedert sich etwa zur Hälfte in Niederungsbereiche, die von Mooren und vor allem von Marschen eingenommen werden, und zur anderen Hälfte in Geestbereiche, die im Drenthe-Stadium der Saale-Kaltzeit angelegt worden sind. Warthestadiales und weichselzeitliches Eis haben unser Gebiet nicht mehr erreicht (Abb. 1). Die äußersten Randlagen des Weichseleises haben zu Oerel eine Mindestentfernung von rd. $75 \mathrm{~km}$ und die des Wartheeises, prägnant markiert durch die Harburger Berge, noch eine Entfernung von rd. $50 \mathrm{~km}$.

Von Gletschereis war das Elbe-Weser-Dreieck zuletzt anläßlich der jüngeren Vereisungsphase des DrentheStadiums, während des Drenthe-2-Vorstoßes, bedeckt. Davon zeugen zwei markante Höhenzüge, die nach WOLDSTEDT (1950) als Altenwalder Staffel und als Lamstedter Staffel bezeichnet werden (Abb. 1). Die Altenwalder Staffel als äußerste Randlage des Drenthe-2-Eises ist im nördlichen Teil (Bereich Altenwalde-Cuxhaven) als Stauchmoräne (SINDOwSKI 1969) und in ihrer südlichen Fortsetzung als Satzmoräne aus ungestörten Schmelzwasserablagerungen ausgebildet. Südlich Bremerhaven tritt sie morphologisch nicht in Erscheinung. Die Drenthe-2-zeitliche Eisbedeckung ist dort jedoch durch die Verbreitung der entsprechenden Grundmoräne nachgewiesen worden (LADE 1980).

Morphologisch ausgeprägter als die Altenwalder Staffel zeigt sich die Lamstedter Staffel, die durchgehend als Stauchmoräne ausgebildet ist. Zunächst N-S-verlaufend, biegt sie ca. $15 \mathrm{~km}$ südlich Oerel nach Südosten um, quert die Oste-Niederung und setzt sich in der Rotenburger Geest fort. Die Stauchenergie muß im nördlichen Bereich der Lamstedter Staffel z. T. beträchtlich gewesen sein, da dort stellenweise alttertiäre Tone, z. B. Eozän bei Lamstedt-Rahden und Hemmoor-Heeßel, an die Oberfläche gelangt sind.

Die Frage nach der Reihenfolge der Entstehung ist noch nicht endgültig beantwortet. Vermutlich ist die Altenwalder Staffel die ältere Randlage, und die Lamstedter Staffel wurde anschließend nach einem partiellen Eisrückzug aufgestaucht (HÖFLE \& LADE 1983).

Grundmoräne des Drenthe-2-Vorstoßes findet sich flächenhaft östlich von Oerel in der Stader Geest, dagegen entlang der beiden Staffeln und zwischen ihnen nur lückenhaft. Im unverwitterten Zustand, d. h. als Geschiebemergel, ist sie sowohl im Elbe-WeserDreieck als auch im Hamburger Raum („Niendorfer Moräne”) durch die Führung von vielen Kreide- und Flint-Geschieben sowie durch einen hohen-Kalkgehalt von meist $20 \%$ und mehr gekennzeichnet. In dieser typischen Ausbildung ist sie nicht verwechselbar, weder mit der älteren Drenthe-1- noch mit der ElsterGrundmoräne. Dies zeigte sich beispielhaft an einem Schlüsselprofil für das norddeutsche Quartär im ehemaligen Kreide-Steinbruch von Hemmor (EHLERS 1979; HÖFLE \& SCHLENKER 1979). Dort waren auch Drenthe-1- und Elster-Grundmoräne durch ihre Farbtöne und Korngrößenzusammensetzung gut zu unterscheiden. Die Unterscheidungskriterien wurden jüngst bei zahlreichen Bohrungen im Bereich der Altenwalder Staffel bestätigt (LADE 1985): Drenthe-1Moräne mit braunen bis gelbbraunen Tönen und gleichbleibender Korngrößenzusammensetzung, dagegen Elster-Moräne mit olivgrauen und dunkleren Tönen und heterogener Korngrößenzusammensetzung.

\subsection{Oerel und Umgebung}

Der Ort Oerel liegt am Fuße des Osthanges der Lamstedter Staffel. Direkt östlich benachbart erstreckt sich die hier ca. $5 \mathrm{~km}$ breite Oste-Niederung. Die Eisrandlage wird gut durch die höchsten Erhebungen der lokalen Umgebung, durch den Hohen Oerel mit + 23,1 m NN und den Acker-Berg mit + 36,7 m NN markiert (s. u. und Abb. 2). Zur benachbarten OsteNiederung hin bewegen sich die Niveauunterschiede im allgemeinen um $15 \mathrm{~m}$, erreichen aber maximal am Acker-Berg rd. $30 \mathrm{~m}$.

Entsprechend dem durchgehenden Stauchcharakter der Lamstedter Staffel sind in Oerel und seiner Umgebung ebenfalls Schichtstörungen zu beobachten. So sind z. B. ca. $5 \mathrm{~km}$ SSE bei Basdahl, ebenfalls am Osthang der Staffel gelegen, Lauenburger Ton und elsterzeitliche Grundmoräne hochgestaucht. Etwa $1 \mathrm{~km} \mathrm{SE}$ der Ortslage Oerel erhebt sich die markante Kuppe des Hohen Oerel, der im Kern aus Drenthe-2-Geschiebemergel (Kalkgehalt: 20,4\%) in der Farbe gelblichbraun-gelblichgrau ( = 10 YR 5/4 - 5 Y 7/2 nach ROCK-COLOR CHART) besteht, hochgestaucht ist und als Härtling herauspräpariert wurde (LADE 1980). Bezeichnend dafür ist die Lage einer alten Mergelkuhle in der Kuppe des Hohen Oerel. Geschiebemergel als Naturdünger ist ebenfalls bei $\mathrm{OE} 36$ abgebaut worden (Der Schießstand auf Abb. 3 liegt in einer Mergelkuhle). Hier zeigt Abb. 4 eine förmliche Faltung der Grundmoräne. Als Geschiebemergelschuppe muß die Geesthalbinsel Fahrenkamp betrachtet werden, auf der Profil 4 mit OE 55 endet (Abb. 8). An all diesen Stauchlokalitäten hat das Drenthe-2-Eis seine eigene Moräne nachträglich gestört.

Einbezogen in die Stauchungen sind auch Schmelzwasserablagerungen, zumindest die am Osthang des Acker-Berges (Abb. 7). Ob das auch für die etwas weiter im S und SE gelegenen Sandgebiete (OE 7-6 


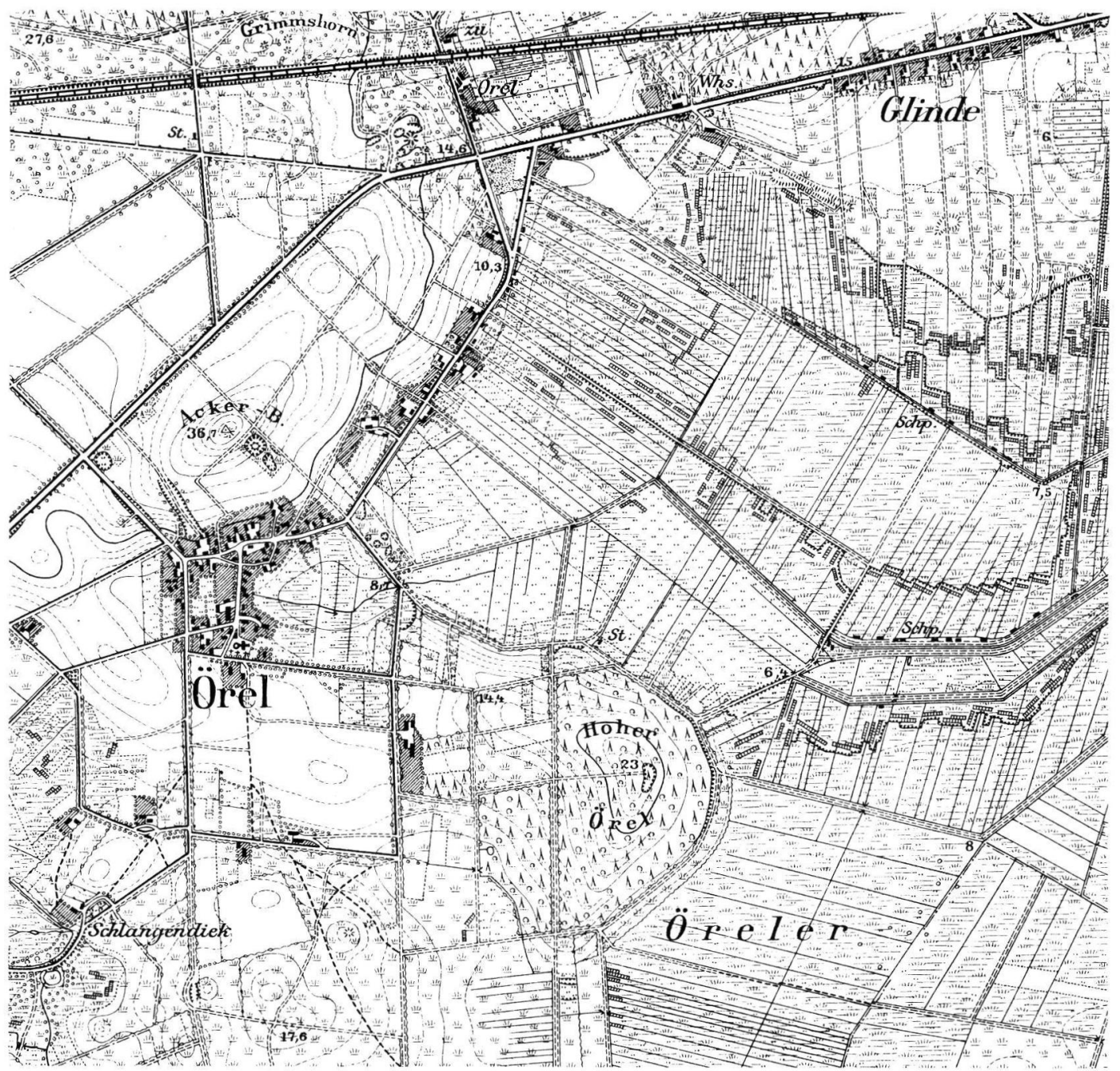

Kartengrundlage: Preußische Landesaufnahme 1:25000, 2520 (1899).

Herausgegeben von der Preußischen Landesaufnahme und vom

Niedersächsischen Landesverwaltungsamt - Landesvermessung -.

Vervielfältigt mit Erlaubnis des Niedersächsischen Landesverwaltungs amtes - Landesvermessung - B5 - 660/85.

Abb. 2: Ausschnitt des Bereichs um Oerel aus der TK 25, Blatt 2520 Bremervörde (Erstausgabe von 1899) Maßstab 1:25000.

in Abb. 6, OE 28-29 in Abb. 4) gilt, läßt sich mangels Aufschlüssen nicht sagen. Möglicherweise ist dort das Stauchungsausmaß erheblich geringer, worauf Stauchungsbeobachtungen und -messungen in einer Sandgrube am nördlichen Fuße des Hohen Oerel hinweisen (LADE 1980). Geringfügig gestaucht zu sein scheinen auch die Beckensedimente am Osthang des Acker-Berges. Auf die Frage, ob die Lagerungsverhältnisse unter der Hohlform auch stauchungsbedingt sind, wird im Kapitel 3 eingegangen.

Zu Petrographie und Farbe der oben genannten glazialen Sedimente sei noch erwähnt, daß die Schmelz- wasserablagerungen überwiegend als Mittel- und Grobsande und die Beckenablagerungen überwiegend als glimmerhaltige Feinsande und - untergeordnet - Schluffe ausgebildet waren. Im Drenthe-2Geschiebemergel gab es neben den vorherrschenden gelblichbraunen bis gelblichgrauen Farbtönen im basalen Bereich häufig olivgraue (5 Y 4/1), wie sie auch von HÖFLE aus Hemmor (1979) und von Blatt 2624 Hollenstedt der GK 25 (HÖLLE 1982) sowie von MEYER auf Blatt 2524 Buxtehude der GK 25 (MEYER 1982) beschrieben worden sind. 
Im tiefsten Aufschluß, OE 61 (Abb. 10), sind ältere Schichten als bisher zitiert erbohrt worden. Zwischen $35 \mathrm{~m}$ und $38 \mathrm{~m}$ lagert ein dunkler kalkhaltiger Ton, der wegen seiner faziellen Ausbildung und seiner Lage am Rande einer elsterzeitlichen Rinne (KUSTER \& MEYER 1979) dem Lauenburger Komplex zugeordnet wurde. Dann folgen Schmelzwassersande und -kiese und anschließend ein $2 \mathrm{~m}$ mächtiger Geschiebemergel, der wegen seiner Tiefenlage, seiner olivgrauen Farbe (5 Y 3/2) und der Überlagerung durch vermutlichen Lauenburger Ton in die Elster-Kaltzeit gestellt wurde. Allerdings bestätigen dies die Feinkiesanalysen (Zählbereich: 4-6,3 mm) der Moräne und der liegenden Schmelzwasserschichten, gemessen an den feinkiesanalytischen Standardergebnissen aus dem Bereich der Altenwalder Staffel (LADE 1985) nicht eindeutig. Die Ergebnisse von Oerel mit viel Kristallin, viel Flint und wenig Quarz zeigen kein typisches Elster-Spektrum, sondern befinden sich im Überlagerungsbereich von Elster und Drenthe-1 (Moräne) bzw. im Drenthe-1-Bereich (Schmelzwasserkies). Daher bleibt die stratigraphische Einstufung von Ton, Moräne und Schmelzwasserschichten vorläufig unsicher.

Sicher ist dagegen das Alter von Schmelzwasserschichten und Moräne im Hangenden des Tones. Neben den makroskopischen Befunden bestätigt dies die Feinkiesanalyse der Schmelzwasserschichten: viel Flint, wenig Kristallin, sehr wenig Quarz (1 Korn) und mehr Kreide- als paläozoische Kalke (aus der Moräne konnte aus bohrtechnischen Gründen keine Probe genommen werden).

Als letzte saalezeitliche Schicht wurden Sande mit unterschiedlichen Kiesanteilen und z. T. tonig-schluffigkiesigen Beimengungen angetroffen. Sie bilden fast überall das Liegende der jeweils untersten organogenen Schicht und - sofern erbohrt — das Hangende der Drenthe-2-Moräne. Genetisch sind es überwiegend niveofluviatile Sande, die vom Geesthang gekommen und in der Hohlform vermutlich fluviatil weitertransportiert worden sind. Bei tonig-schluffigkiesiger Ausbildung dürfte es sich um Fließerden aus Grundmoränenmaterial handeln.

\section{Die Hohlform von Oerel}

Die Hohlform von Oerel ist eine buchtförmige und vermoorte Niederung, die von höheren Geestgebieten umrahmt wird und nach Osten einen schmalen Entwässerungsdurchlaß zur Oste-Niederung hat (Abb. 2). Durch die umlaufenden Straßen wird sie auf Abb. 3 gut begrenzt.

Um die geologischen Verhältnisse der Bucht und der benachbarten Geest zu erkunden, wurden 5 Profilschnitte gelegt. Profilschnitt 2 (Abb. 6) sollte prüfen, ob der Entwässerungsdurchlaß einen Überlauf darstellt oder ob dort in größerer Tiefe schon entwässert wurde. Der Profilschnitt 1 (Abb. 4) quert als einziger die Niederung mittig, während sich die Profilschnitte 3, 4 und 5 (Abb. 7-9) am nördlichen Rand befinden. Aus den restlichen Niederungsbereichen liegen keine Aufschlüsse vor, doch haben Baugrundbohrungen für das Rathaus am westlichen Rand der Niederung ( $\mathrm{Abb} .3$, in Höhe Ortsname Oerel) keine organogene Schichten angetroffen.

Die Untersuchungsergebnisse zeigen eine fossile Hohlform an, die - abgesehen von der dünnen holozänen Torfauflage - eine Folge von maximal 5 organogenen Schichten mit zwischengelagerten Sanden beherbergt. Die Pollenanalysen ergaben, daß stets die unteren 3 Schichten in der Eem-Warmzeit, im Brörup- und im Odderade-Interstadial gebildet wurden (vgl. 4.2.-4.4.) Sie sind unter fast der gesamten Niederung gemeinsam vorhanden und erstrecken sich über maximal $500 \mathrm{~m}$ (OE 39-OE 26, Abb. 4). Dagegen repräsentieren die beiden oberen der 5 organogenen Schichten neu beschriebene Interstadiale (s. u. und 4.5.-4.6.) und beschränken sich auf den nördlichen Teil der Niederung, in dem die Hohlform am tiefsten ist. Hier wie auch im restlichen Teil werden durch $\mathrm{Abb}$. 4 Lagerungsstrukturen angezeigt, die für die Hohlform eine glaziäre Entstehungsursache annehmen lassen.

Durch das eemzeitliche Alter der untersten organogenen Schicht (Beginn in OE 22: Kiefern-Eichenmischwald-Zeit, Eem III nach BEHRE 1962) ist die Entstehung der Hohlform in der Saale-Kaltzeit gesichert. In Frage kommen eine Pingo-bedingte Genese in der Periglazialzeit des Warthe-Stadiums oder Entstehungsweisen, die mittelbar oder unmittelbar auf das letzte glaziäre Geschehen am Ort während der Drenthe-2-Vereisung zurückzuführen sind. Die Pingo-bedingte Genese ist nicht wahrscheinlich, weil ein entsprechender Wall fehlt, vor allem aber, weil dann damit gerechnet werden muß, daß die Grundmoräne ausgeräumt ist. Sonst wäre das Massendefizit schwer erklärbar.

Es bleiben Entstehungsmöglichkeiten im Zusammenhang mit Gletschereis: Auskolkung und Toteis. Da auch bei einer Auskolkung erwartet werden muß, daß die Grundmoräne erodiert ist, wird stattdessen eine Genese wahrscheinlich, bei der Toteis die entscheidende Rolle spielt. Möglicherweise war die Hohlform nach Totfallen des Drenthe-2-Eises plombiert und bildete sich später im Zuge des Abschmelzprozesses heraus. Das unruhige Basisrelief könnte dann durch Stauchung während des Lamstedter Vorstoßes erklärt werden. Dafür spricht die Lage des Hohlformtiefsten am Fuße des gestauchten Hanges, also gerade dort, wo der Hauptdruck zwischen Eis und mineralischem 


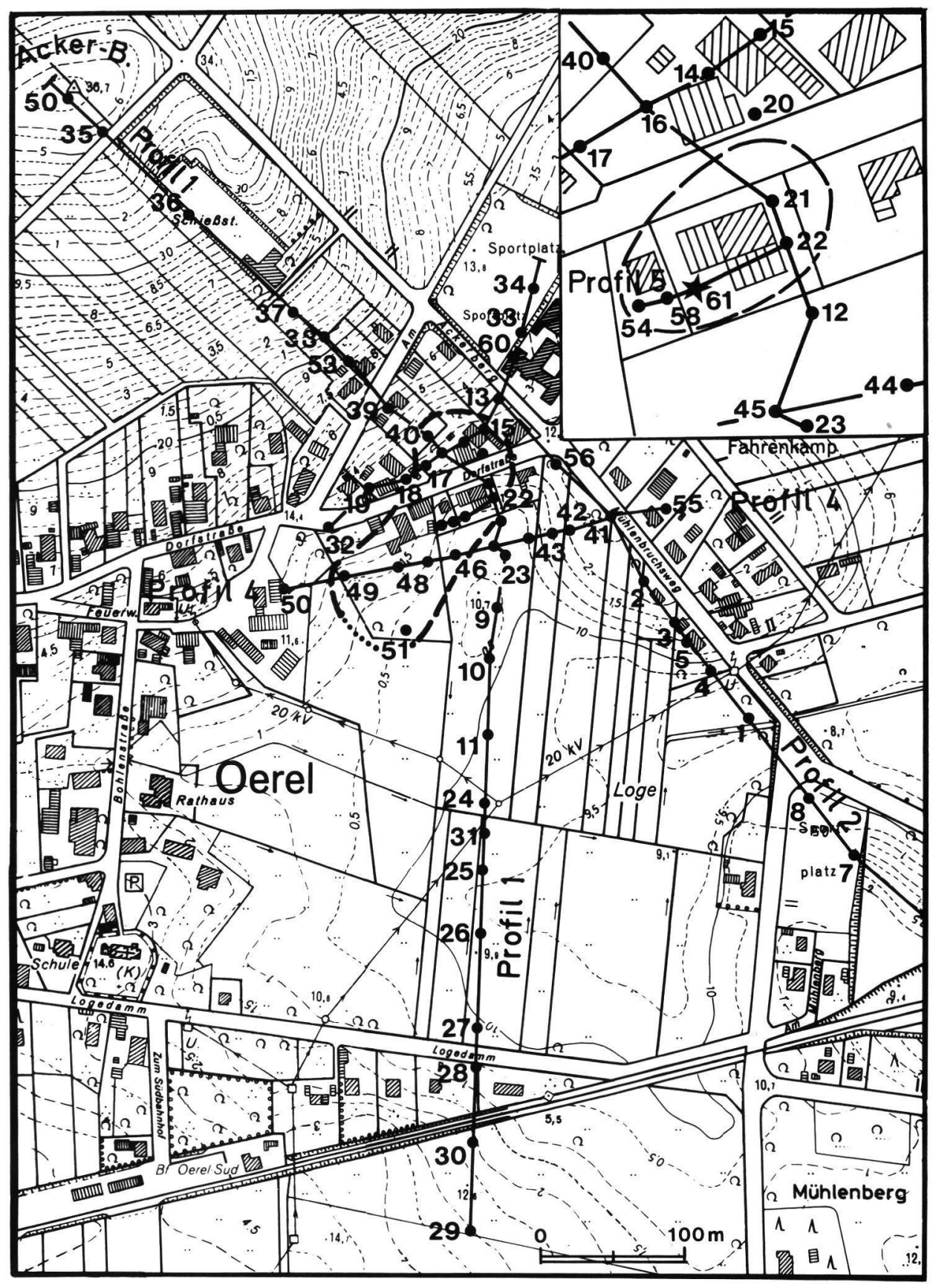

Abb. 3: Lage der Profilschnitte und Bohrungen in Oerel.

Hauptkarte: Maßstab 1:6000, umgrenzt ist die Ausdehnung des Oerel-Interstadials Ausschnittkarte rechts oben: Maßstab 1:2000, umgrenzt ist die Ausdehnung des Glinde-Interstadials. Vervielfältigungserlaubnis unter AZ AR 23/85 am 05. 03. 85 erteilt durch das Katasteramt Bremervörde.

Hindernis entstand und sich aktives Eis in den Untergrund pressen konnte. Andererseits kann das Abtauchen der Grundmoräne als Sackungsstruktur gedeutet werden, wonach unter der Moräne verschüttetes Tot- eis post-Drenthe-zeitlich abtaute und die Deckschichten nachsacken ließ. Das unruhige Relief der Moränenoberfläche könnte auf unterschiedliche Toteismächtigkeiten zurückgeführt werden. 
PROFIL 1

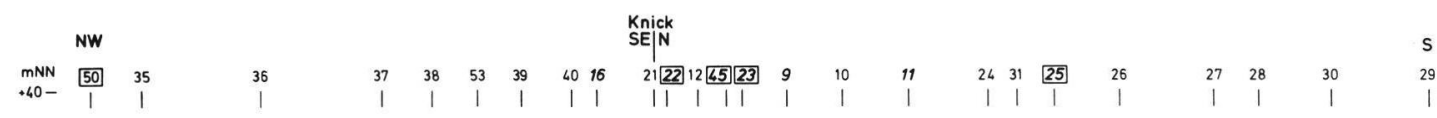

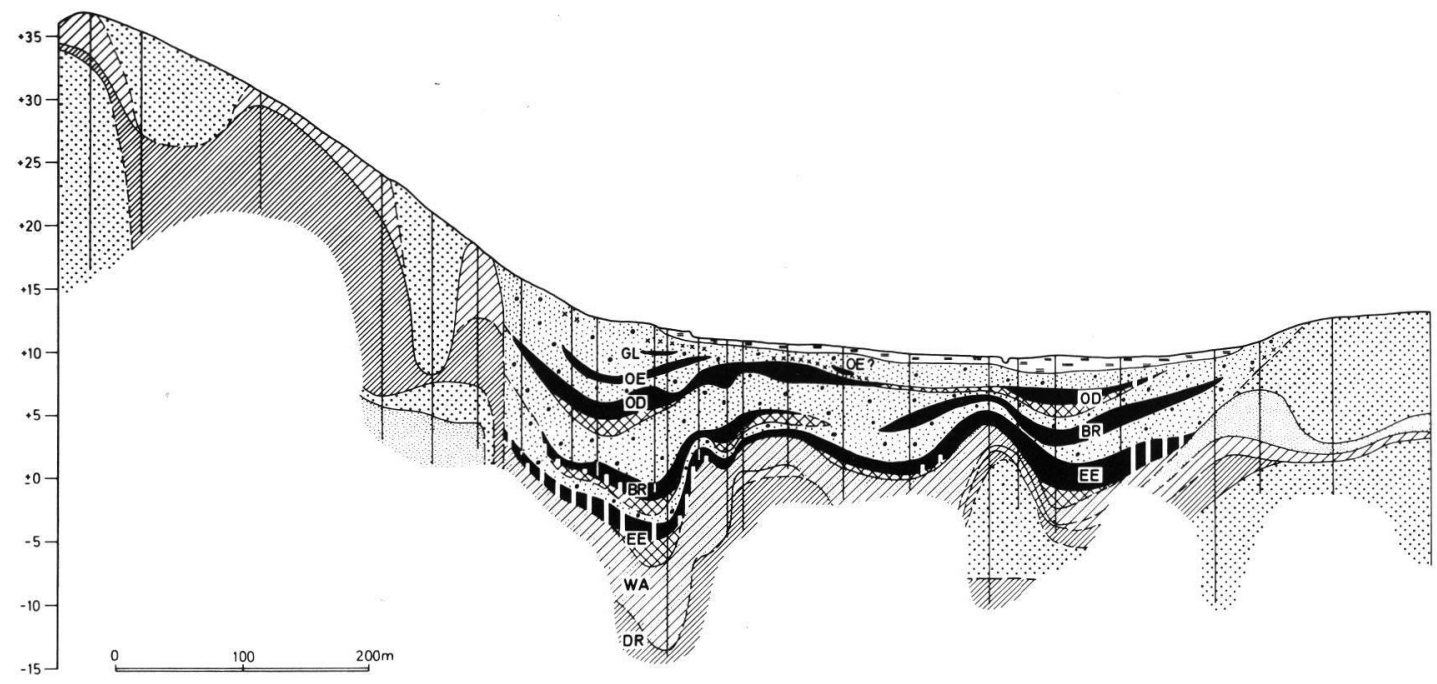

Abb. 4: Profilschnitt 1, Überhöhung 10:1; zur Lage vgl. Abb. 3, Legende vgl. Abb. 5;

$\mathrm{DR}=$ Drenthe, $\mathrm{WA}=$ Warthe, $\mathrm{EE}=\mathrm{Eem}, \mathrm{BR}=$ Brörup, $\mathrm{OD}=$ Odderade, $\mathrm{OE}=$ Oerel, $\mathrm{GL}=$ Glinde .

Wo das Toteis auch gelegen haben mag: Bereits im Warthe-Stadium kam es zu reliefbedingten Umlagerungen, möglicherweise in eine damals schon existierende Hohlform hinein. Darauf weist die Mächtigkeitszunahme der entsprechenden Ablagerungen unter dem tiefsten Bereich der eemzeitlichen Hohlform hin (Abb. 4). Ablagerungen eines zwischen Drenthe2-Vereisung und Warthe-Stadium postulierten Interglazials, der Treene-Warmzeit (PICARD 1959), die die Hohlform bereits damals hätte entstehen lassen und organogene Schichten hätte hinterlassen müssen, wurden an keiner Stelle beobachtet.
Spätestens zu Beginn der Eem-Warmzeit muß die Hohlform als solche vorhanden gewesen sein. In OE 22 begann bereits im Eem III, in OE 61 im Eem IVa die limnische Sedimentation. Älteres Eem und SaaleSpätglazial wurden bisher nicht festgestellt, könnten aber $z$. B. bei OE 47, wo die Torfunterkante tiefer als in OE 22 und OE 61 liegt (s. u.), ausgebildet sein.

Die eemzeitlichen Mudden in OE 22 und OE 61 führen nur relativ wenig humoses Material und sind lagenweise dunkelbraun (5 YR 2/2), ansonsten gräulicholivgrün bis olivgrau (5 GY 3/2-5 Y 3/2) gefärbt.
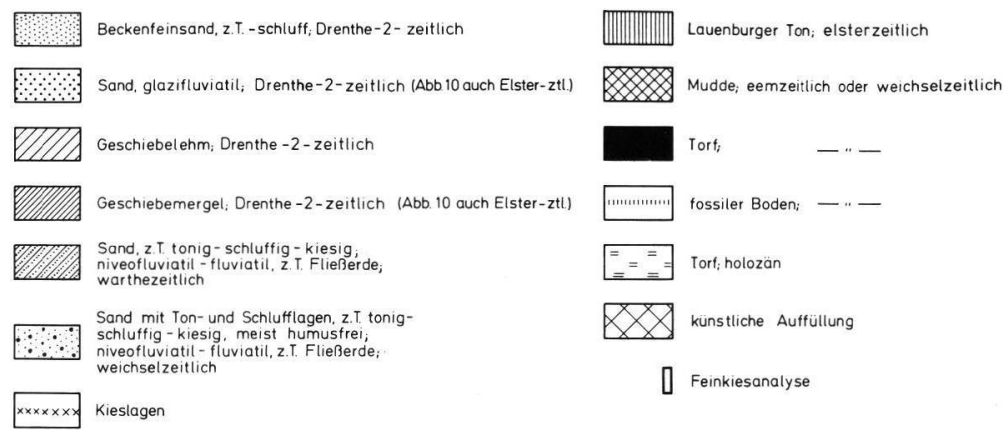

50 verrohrte Bohrung

1622 organogene Schichten datiert

Abb. 5: Legende zu den Profilschnitten. 


\section{PROFIL 2}

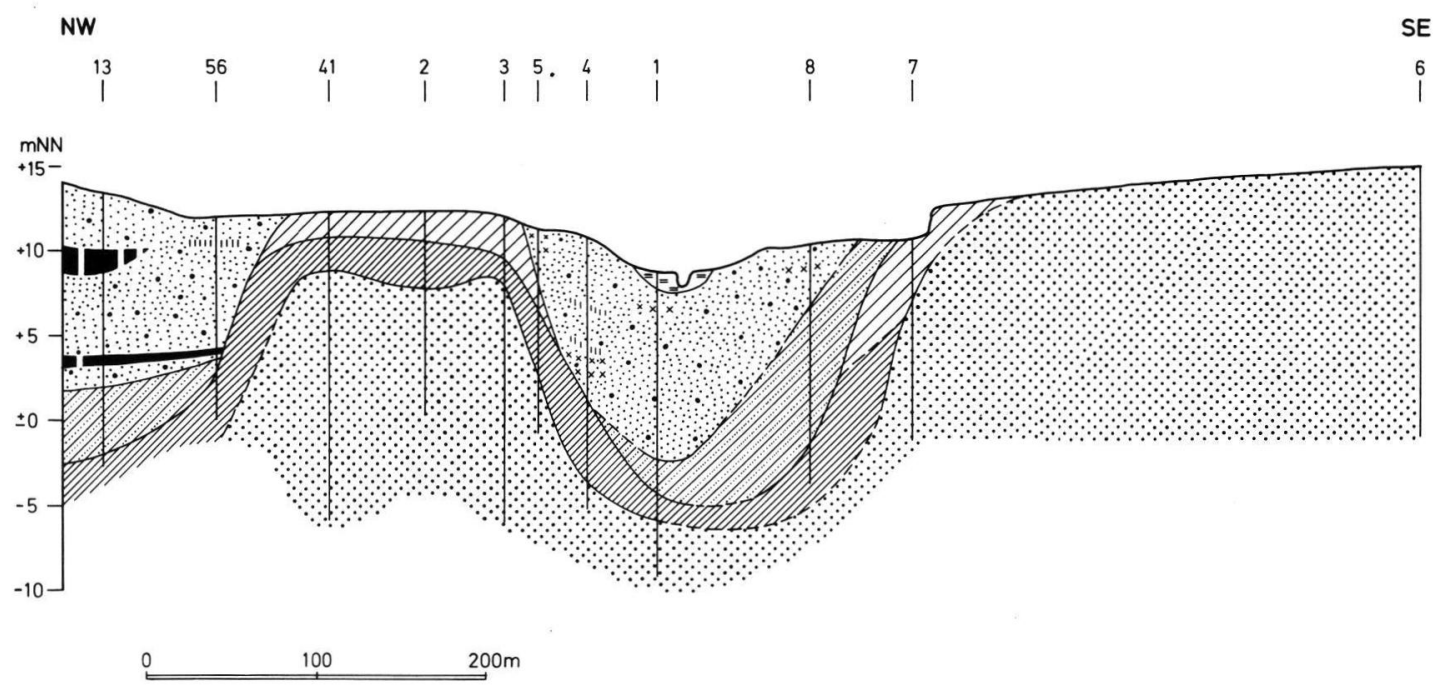

Abb. 6: Profilschnitt 2, Überhöhung 10:1; zur Lage vgl. Abb. 3, Legende vgl. Abb. 5.

Kalkgehalt ist meistens vorhanden, nur im unteren Teil gibt es kalkfreie Lagen. Nach oben nimmt der Kalkgehalt und gleichzeitig die Führung von Ostracodenschalen generell zu. Der stärkste Kalkgehalt in OE 61 wurde im obersten Teil der Mudde (cm 1684 bis 1760) festgestellt, dem fast genau der Abschnitt Eem V entspricht. Lieferant des Kalkes war vermutlich die kreidekalkreiche Moräne des angrenzenden Hanges.

Gegen Ende von Eem V (OE 9), spätestens in Eem VI (z. B. in OE 61), beginnt in der Hohlform von Oerel flächenhaft Niedermoortorf zu wachsen. Der damalige Grundwasserspiegel lag, abgeschätzt an der Torfunterkante von OE 9, bei rd. +3,15 m NN. Der gleichalte Torf an anderen Stellen der Hohlform liegt, bezogen auf die datierten Strecken, um bis zu 7,4 m (OE 61), nach OE 47 (ohne Datierung korreliert) sogar um $9 \mathrm{~m}$ tiefer. Dieser Befund, erkennbar an den Lagerungsverhältnissen der organogenen Schichten, hat seine Ursachen nicht mehr in Toteissackungen, sondern in Setzungen des Sediments. Da in OE 9 unter dem Eemtorf setzungsfähiges organisches Material nicht mehr vorkommt und da davon ausgegangen werden kann, daß gleichalte Niedermoortorfe niveaugleich wachsen, sind die angegebenen Sackungsbeträge absolute Beträge. Das heißt, die limnische Sedimentation hatte in Eem V/VI ein Niveau von etwa $+3,15 \mathrm{~m} \mathrm{NN}$ erreicht.

Zeugen trockener Standorte wurden als Bodenrelikt nur in $\mathrm{OE} 42$ in Form eines $\mathrm{B}_{\mathrm{hs}}$-Horizontes beobachtet (Abb. 8).
Der Beginn der W e i chse $1-\mathrm{Kal}$ t z e it wird in Oerel im allgemeinen durch die erste posteemzeitliche Sandschicht angezeigt, abgelagert im $\mathrm{He} \mathrm{r}$ $\mathrm{n}$ i $\mathrm{n} \mathrm{g}-\mathrm{S} \mathrm{t}$ a $\mathrm{d}$ i a $\mathrm{l}$. Nimmt man die vegetationsgeschichtliche Definition der Grenze Eem/Weichsel (vgl. S. 25), dann reicht der ausklingende Torf jedoch noch bis in das frühe Herning-Stadial hinein, bei $\mathrm{OE}$ 61 von $\mathrm{cm} 1635$ bis 1609. Nach der Frühweichselgliederung von MENKE \& TYNNI (1984), der in dieser Arbeit gefolgt wird, ist es der Abschnitt WF I (vgl. Tab. 1). Die Sande wurden genetisch als überwiegend niveofluviatil eingestuft, können aber in größerer Entfernung vom Geesthang fluviatil weitertransportiert worden sein. Petrographisch sind es Mittelsande sowie daneben Fein- und Grobsande mit wenigen umgelagerten humosen Resten und geringem Anteil an Pflanzenhäcksel. Eine Ausnahme bildet der Bereich um OE 39 (Abb. 4). Bedingt durch die Nachbarschaft zum Geschiebemergel, wechseln mit dem Sand Tone und Schluffe sowie, als Gemisch aller Korngrößen, Fließerden ab. Die Stärken dieser stadialen Schicht betragen meist nicht mehr als $2 \mathrm{~m}$, in einem Falle jedoch 6,5 m (OE 47). Gegen Ende des Herning-Stadials, vermutlich bedingt durch die sich ausbreitende Bodenvegetation und die damit einhergehende Sedimentationsberuhigung, lagern sich in den tieferen Hohlformbereichen Ton- und Schluffmudden mit wenig organischen Bestandteilen ab (vgl. 4.3 und Abb. 13).

Im nachfolgenden B r ör u p - In t e r s t a d i a l, WF II, hält die limnische Sedimentation in den tieferen Hohlformbereichen zunächst an. Die nach vegeta- 
PROFIL 3

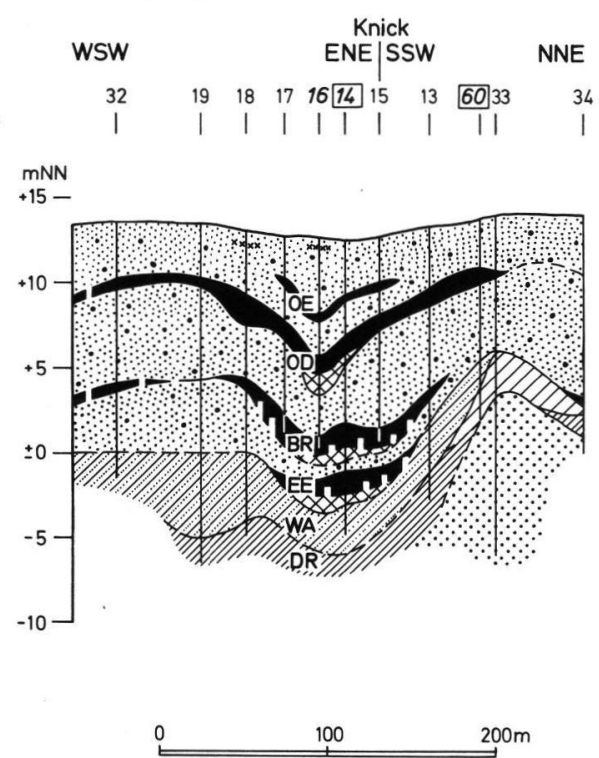

Abb. 7: Profilschnitt 3, Überhöhung $10: 1$; zur Lage vgl. Abb. 3, Legende vgl. Abb. 5

tionsgeschichtlichen Kriterien festgesetzte Grenze Stadial/Interstadial liegt in OE 61 bei $\mathrm{cm} 1455$ noch in der Mudde. Den Hauptanteil der Brörup-Schichten machen allerdings Torfe aus, wie sie bisher auch an 8 weiteren Lokalitäten im Elbe-Weser-Dreieck festgestellt wurden (BEHRE 1974; LADE 1980; HAGEDORN \& LADE 1982).

Bodenrelikte, die nach ihrer Tiefenlage dem Brörup zugeordnet werden können, wurden nur in OE 43 beobachtet (Abb. 4). Es waren ein $B_{s}$ - und hangend ein weißer $\mathrm{A}_{\mathrm{e}}$-Horizont, dem ein $5 \mathrm{~cm}$ starkes Humussediment auflag (selektiv umgelagerter $A_{h}$; vgl. LADE \& HAGEDORN 1982).

Über dem Brörup liegen fluviatil-niveofluviatile Feinund Mittelsande und, mit geringeren Anteilen, Grobsande, abgelagert im Rederstall-Stadia l, WF III. Im Gegensatz zu den Herning-stadialen und auch zu den hangenden stadialen Sanden zeichnen sich diese durch einen erheblich höheren Anteil an umgelagertem Pflanzenhäcksel und feinen humosen Lagen aus. Dadurch hatten sie bei den Geländeuntersuchungen nahezu stratigraphischen Leitcharakter. Wie schon im Herning-Stadial entstanden auch jetzt gegen Ende dieses Stadials Ton- und Schluffmudden mit geringen organischen Beimengungen. Die Gesamtmächtigkeiten sind allgemein höher als im vorangegangenen Stadial und liegen meist bei $3-4 \mathrm{~m}$, maximal sind es $5,65 \mathrm{~m}$ (OE 22).
PROFIL 4

WSW

ENE

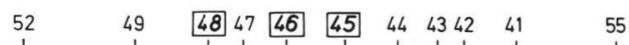

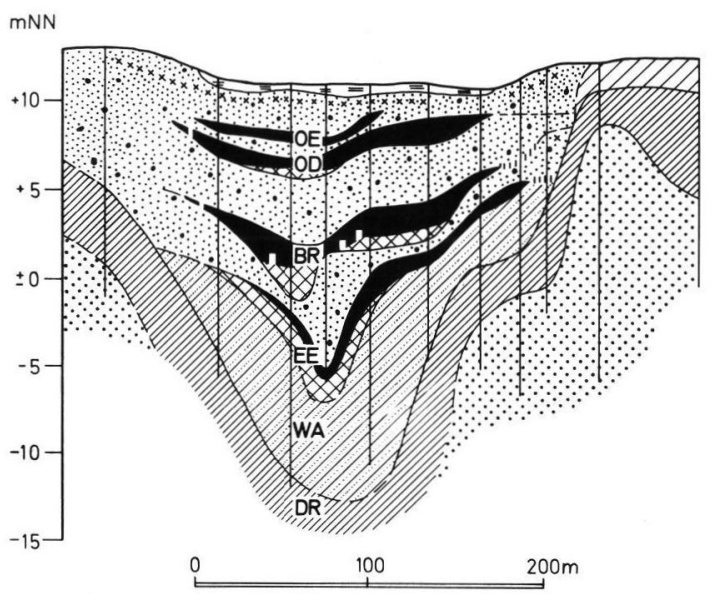

Abb. 8: Profilschnitt 4, Überhöhung $10: 1$; zur Lage vgl. Abb. 3, Legende vgl. Abb. 5.

Dieser Befund, daß im Rederstall stärkere Schichten als im Herning abgelagert wurden, beschränkt sich nicht auf die Lokalität Oerel. An 3 weiteren Hohlformen im Elbe-Weser-Dreieck mit nachgewiesenen Brörup- und Odderade-Vorkommen, bei Appeln (auf Blatt 2519 Frelsdorf der TK 25; vgl. LADE 1980), bei Abbenseth (Blatt 2420 Ebersdorf der TK 25) und bei Krempel (Blatt 2218 Wanna der TK 25; vgl. LADE \& HAGEDORN 1982) sowie bei Rederstall in SchleswigHolstein (MENKE \& TYNNI 1984) zeigten sich die gleichen Relationen. Vielleicht ist dies durch eine unterschiedlich lange Zeitdauer der Stadiale, wahrscheinlicher aber klimatisch bedingt, wonach im Herning die Abkühlung nicht so stark und die Vegetation nicht so lange gelichtet war wie im Rederstall.

Die gegen Ende des Rederstall-Stadials einsetzende limnische Sedimentation hielt im nachfolgenden Od d e r a d e - Inter s t a d i a l, WF IV, bei OE 61 noch kurze Zeit an (vgl. 4.4 und Abb. 13). Dann bildete sich bis Ende dieses Interstadials flächenhaft ein Torf. Außerhalb davon wurde bei OE 56 ein fossiler Boden entdeckt, der dieser Zeit entsprechen könnte (Abb. 6). Es handelt sich dabei um einen jeweils schwach ausgebildeten $A_{h}$ - und $B_{s}$-Horizont.

Für die Abschätzung des absoluten Sackungsbetrages vor und nach dem Odderade im Zentrum der Hohlform steht mit dem Odderade-Torf von OE 60 eine sackungsfreie Bezugsschicht zur Verfügung, da an 
dieser Stelle liegend keine weitere organogene Schicht angetroffen wurde. Bezogen auf $\mathrm{OE} 61$ beträgt die Differenz der Torfunterkanten $4,1 \mathrm{~m}$, d. h. postOdderade ist bei OE 61 die Schichtenfolge von Eem bis Odderade um diesen Betrag gesackt, bei OE 16 sogar um 5,05 m. Als Sackungsbetrag prä-Odderade errechnen sich bei OE $613,3 \mathrm{~m}(=7,4 \mathrm{~m}$ im Eem minus $4,1 \mathrm{~m}$ ).

Gemessen an der Torfunterkante bei OE 60 lag das Oberflächenniveau zu Beginn des Odderade im nördlichen Teil der Bucht bei etwa $+10 \mathrm{~m} \mathrm{NN}, \mathrm{d}$. h. in einer der heutigen vergleichbaren Höhe. Wenn nachfolgend erneut und letztlich ohne Geländeerhöhung sedimentiert wurde, so nur nach weiteren und flächenhaften Sackungen.

Auf das Odderade-Interstadial folgt eine nächste kalte Phase, die nach MENKE \& TYNNI (1984) S c h a $1 \mathrm{k}$ $\mathrm{h}$ ol z-St a d i a l genannt und noch ins Frühweichsel (,WF V”) gestellt wurde, die wir aber im Gegensatz zu beiden und in Anlehnung an den Vorschlag von CHALINE \& JERZ (1984) an den Beginn des Weichsel-Pleniglazials stellen: WP I (vgl. Tab. 1). Abgelagert wurden überwiegend Fein- und daneben Mittelsande mit sehr geringen organischen Anteilen. Dort, wo die Sande durch das hangende Interstadial begrenzt sind, liegen die Schichtstärken zwischen einigen $\mathrm{dm}$ und maximal 1,9 $\mathrm{m}$ (OE 14).

Im nördlichen Teil der Bucht werden diese Sande durch einen weiteren Torf abgedeckt, der eine waldlose Phase darstellt und dessen Mächtigkeit stets unter $1 \mathrm{~m}$ bleibt (bei OE $610,8 \mathrm{~m}$ ). Seine Lagerung zeigt, daß nach seiner Bildung unterschiedlich starke Sackungen abgelaufen sind. Absolute Sackungsbeträge lassen sich nicht berechnen, da im Verbreitungsbereich sich im Liegenden stets setzungsfähige Sedimente befinden. Die relative Differenz beträgt von OE 16 nach OE 14 , bezogen auf Torfunterkanten, $1,07 \mathrm{~m}(2,1 \mathrm{~m}$ bei den ohne Datierung korrelierten Torfen in OE 14 und OE 47). In seiner Verbreitung beschränkt sich dieser Torf bezeichnenderweise auf einen Bereich, der gleichzeitig das Hohlformtiefste begrenzt und Längs- und Querdurchmesser von rd. $200 \mathrm{~m}$ und $100 \mathrm{~m}$ hat (Abb. 3).

Dieser Torf, der bereits bei SELLE \& SCHNEEKLOTH (1965) erwähnt wird, stellt ein eigenständiges Interstadial dar (s. u.); wir benennen es nach dem Fundort mit $\mathrm{O}$ e r e l- I n ter st ad i a l (WP II, vgl. Tab. 1 u. Abb. 5-10).

Über dem Oerel folgt erneut ein Stadial, das sich petrographisch überwiegend durch Fein- und daneben Mittelsande mit wenigen humosen und Pflanzenhäcksel-Lagen darstellt. Die Schichtstärke beträgt maximal $1,5 \mathrm{~m}$ (OE 58). Nach dem $5 \mathrm{~km}$

\section{PROFIL 5}

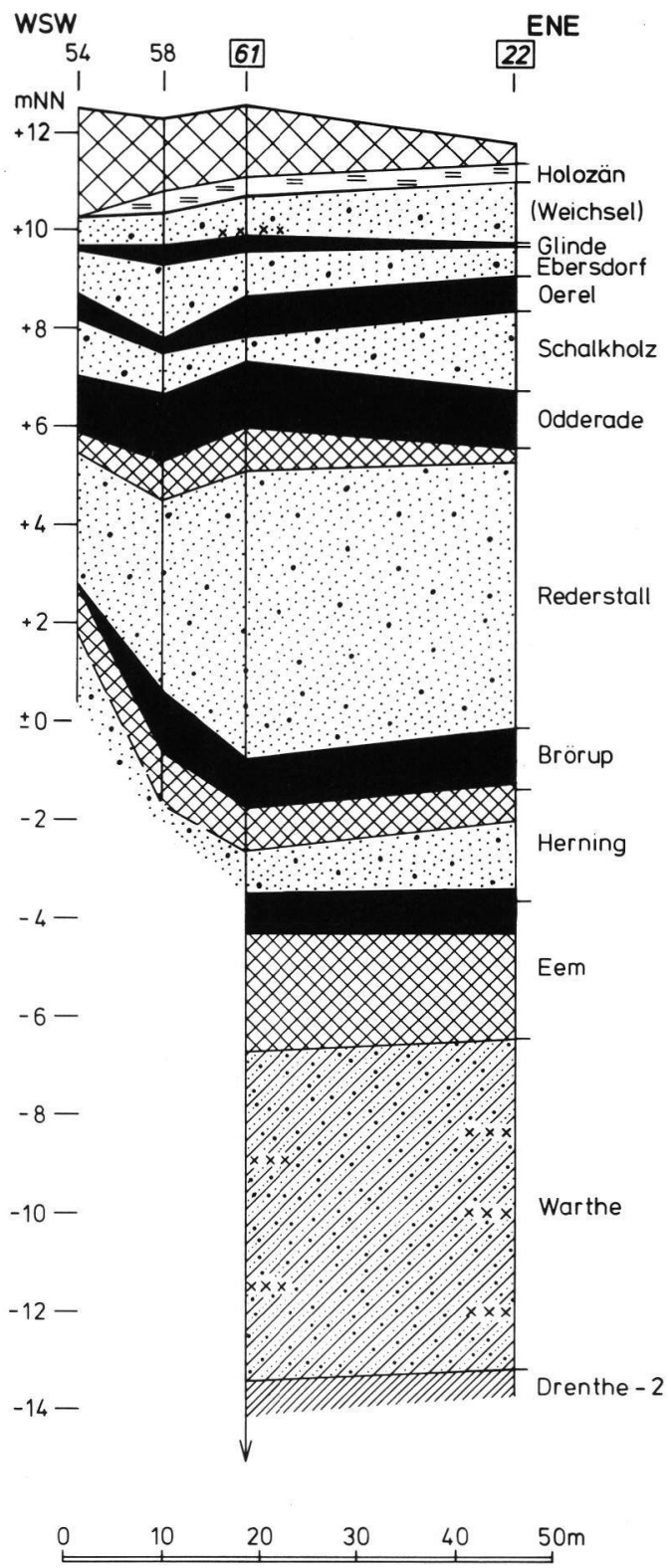

Abb. 9: Profilschnitt 5, Überhöhung 5:1; zur Lage vgl. Abb. 3, Legende vgl. Abb. 5.

nördlich liegenden Ort Ebersdorf bezeichnen wir es mit $\mathrm{E}$ b e r s d o r f - $\mathrm{S}$ t a d i a $\mathrm{l}$ (WP III, vgl. Tab. 1).

Begrenzt werden die Ebersdorf-stadialen Sande nach oben durch einen weiteren weichselzeitlichen Torf, der ebenfalls eine waldlose Phase repräsentiert (vgl. 
OE 61

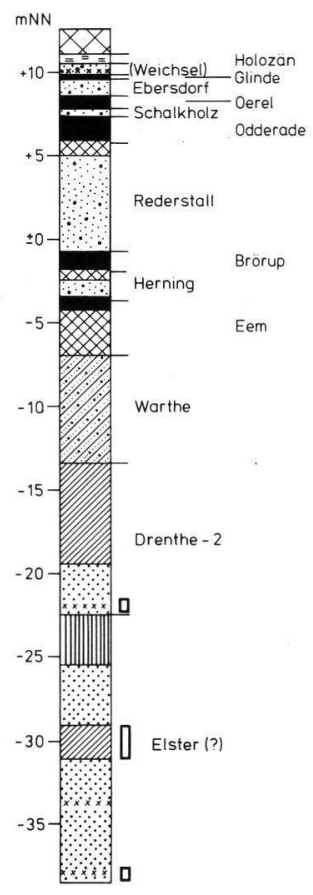

Abb. 10: Das Standardprofil OE 61; gegenüber den Profilschnitten 1-5 geänderter Maßstab! Zur Lage vgl. Abb. 3, Legende vgl. Abb. 5.

S. 28). Er ist maximal 0,23 m stark (OE 61) und befindet sich innerhalb der Verbreitung des Oerel-Torfes, genau: im Zentrum des Hohlformtiefsten innerhalb einer ellipsenförmigen Fläche mit Durchmessern von etwa $65 \mathrm{~m}$ und $35 \mathrm{~m}$ (Abb. 3, rechts oben). Diese Torfschicht ist insgesamt 9 mal erfaßt worden, wenn man die 4 Schlitzsondierungen zur Feststellung des Baugrundes für das Gebäude der Volksbank (direkt nördlich $\mathrm{OE} \mathrm{54-OE} 58$ in $\mathrm{Abb}$. 3) mit berücksichtigt, und wurde in OE 22, OE 59 (in Abb. 3 nicht eingetragen, liegt $5 \mathrm{~m}$ südlich $\mathrm{OE} 61$ ) und $\mathrm{OE} 61$ pollenanalytisch untersucht. Sackungen, deren absolutes Ausmaß sich nicht berechnen läßt, haben auch nach Bildung dieses Torfes stattgefunden. Darauf weisen die relativen Sackungsdifferenzen von maximal 0,4 m zwischen OE 54 und OE 58 (vgl. Abb. 3) hin. Bodenrelikte dieser Schicht wurden nicht beobachtet. Auch dieser Torf stellt ein eigenständiges Interstadial dar (s. u.), und wir benennen es nach dem $2 \mathrm{~km}$ nordöstlich gelegenen Ort Glinde (Abb. 2) mit G 1 i n d e I $\mathrm{t}$ e $\mathrm{r} \mathrm{s} \mathrm{t}$ a d i a 1 (WP IV, vgl. Tab. 1 und Abb. 4,9 u. 10).

Den Abschluß der weichselzeitlichen Sedimente bilden fluviatile bis niveofluviatile Sande aller Korngrößen, oft mit kiesigen Anteilen. Häufig kamen kiesige und tonig-schluffig-kiesige Lagen vor (Abb. 4-10). Bei Ausschachtungsarbeiten für die Probengewinnung bei $\mathrm{OE} 46$ (Versenkung von Kesselrohren von $500 \mathrm{~mm} \varnothing$ ) tauchte in ca. $1 \mathrm{~m}$ unter Geländeoberfläche ein Steinpflaster auf, das genetisch ebenso wie die kiesigen und bindigen Schichten als Fließerde betrachtet wird und vermutlich im späten Pleniglazial zur Ablagerung kam. Schließlich wuchs im Holozän ein bis 1,3 m starker Niedermoortorf, der das Anstehende der Oereler Bucht darstellt (Abb. 4).

An dieser Stelle soll noch einmal zusammengefaßt werden, warum Oerel- und Glinde-Torf stratigraphisch eigenständige Interstadiale innerhalb einer vollständigen Serie sind. Aus geologisch-morphologischer Sicht belegen dies die folgenden Befunde:

a) die Lage in einer Hohlform, in der es keine Anzeichen für nennenswerte Erosion gibt, sondern in der überwiegend akkumuliert wurde und deshalb keine Schichtlücken zu erwarten sind,

b) die Lage der obersten Interstadiale über dem Hohlformtiefsten, also gerade dort, wo wegen der stärksten Anhäufung organogenen Materials und der einhergehenden stärksten Setzungsfähigkeit am ehesten Interstadialbildungen jünger als Odderade zu erwarten sind,

c) die durchgehende Trennung gegenüber den jeweils älteren und jüngeren Interstadialen durch minerogene Sedimente und

d) die flächenhafte Verbreitung beider Torfe übereinander, die ausschließt, daß der oberste Torf eine Wiederholung des darunterliegenden ist.

Im Kap. 4 wird im folgenden gezeigt, daß durch die pollenanalytischen Untersuchungen die Oerel- und Glinde-Interstadiale nicht nur gegenüber Brörup und Odderade, sondern auch untereinander durch unterschiedliche Vegetationsverhältnisse gekennzeichnet sind. Die Gliederung des Weichsel-Frühglazials und des Weichsel-Pleniglazials in Tab. 1 erfolgt demnach mit stratigraphischer und vegetationsgeschichtlicher Absicherung.

Schließlich soll noch auf die Frage eingegangen werden, warum gerade in Oerel nicht nur die beiden großen Interstadiale Brörup und Odderade, sondern zusätzlich 2 jüngere in organogener Form anzutreffen sind.

Aus der vorangegangenen Beschreibung geht hervor, daß die Eembasis im Zentrum der Hohlform sehr tief liegt, bei OE 61 (abzüglich der rezenten Auffüllung) rd. $17,5 \mathrm{~m}=$ rd. $-6,5 \mathrm{~m} \mathrm{NN}=$ rd. $17 \mathrm{~m}$ unter heutigem Grundwasserspiegel $=\mathrm{rd}$. 9,5 $\mathrm{m}$ unter dem eemzeitlichen Grundwasserspiegel. So konnten sich eemzeitlich bei OE 61 erst rd. 9,5 m Mudde und 
anschließend ca. $1,5 \mathrm{~m}$ Torf (heutige Mächtigkeit: $0,75 \mathrm{~m}$, angenommener Sackungsquotient: nur $50 \%$ ), zusammen wenigstens $11 \mathrm{~m}$ setzungsfähiges Material bilden, um anschließend auf insgesamt ca. $25 \%$ seiner ursprünglichen Mächtigkeit komprimiert zu werden. Dieser Befund einer primär tief unter dem Grundwasserspiegel liegenden Hohlformbasis zusammen mit starker Limno- und Torfproduktion und entsprechend starker Setzungsfähigkeit scheint in Oerel die Hauptursache für die Ausbildung aller 4 Interstadiale in organogener Form zu sein. Da zwischenzeitlich im Odderade die Hohlform auf das heutige Niveau angefüllt war, haben sich Oerel- und GlindeTorf nur bilden können, weil die Interstadial- und die Eemschichten noch weiterhin setzungsfähig waren.

Hinzu kommt ein morphologischer Aspekt: Im Profilschnitt 2 (Abb. 6), der die heutige Entwässerungsbahn der Oereler Bucht quert (OE 1), taucht die Grundmoräne ebenfalls ab und zeigt an, daß spätestens ab Eem auch schon hier entwässert wurde (die Grenze Warthe/Weichsel wurde nach dem Vorkommen organischer Sedimentanteile gezogen). Dies ist im übrigen auch über den Bereich OE 13-OE 56 (Abb. 6) möglich gewesen. Auf jeden Fall war die Oereler Hohlform keine rundum geschlossene Senke, sondern hatte Verbindung mit der Vorflut in der Oste-Niederung. Über diese Verbindung könnte eine gewisse Regulierung der von den Hängen gelieferten Fracht stattgefunden haben, wodurch ein Teil in die Oste-Niederung weitertransportiert und damit ein schnelles Auffüllen der Senke verhindert wurde. Darauf weist auch ein scheinbarer Widerspruch hin: die Lage der 4 interstadialen Schichten in enger Nachbarschaft zum relativ steilen Hang des Acker-Berges, also in einem Bereich, der am schnellsten hätte aufgefüllt sein müssen. Die morphologische Zwitterstellung der Oereler Hohlform, weder geschlossene Senke noch offene Rinne, könnte diesen Widerspruch lösen.

\section{Vegetationsablauf und zeitliche Einordnung}

\subsection{Allgemeines zu den botanischen Untersuchungen}

Von den zahlreichen Bohrungen und Sondierungen von Oerel wurden mehrere Profile pollenanalytisch untersucht, in den meisten Fällen allerdings nur so weit, wie es zur sicheren Korrelierung der organischen Schichten notwendig war. Detailliert bearbeitet wurden das vollständigste Profil OE 61, das im folgenden beschrieben wird, sowie ein Teil des Profils OE 46.

Die Pollenerhaltung war in der Regel gut; die Aufbereitung erfolgte im ganzen Profil mit Hilfe einer HFBehandlung und anschließender Azetolyse. Im Eem und in den beiden bewaldeten Interstadialen wurden mindestens 500 Baumpollen pro Spektrum gezählt, in den beiden unbewaldeten Interstadialen mindestens 500 Pollen insgesamt. Die Pollenzahlen der Einzelproben finden sich in den Diagrammen. Soweit außerhalb der Zählgänge zusätzliche Pollentypen gefunden wurden, sind sie mit I für einzelnes und + für mehrfaches Vorkommen in den Diagrammen vermerkt. Um eine gute Vergleichbarkeit zu ermöglichen, wurden alle Pollendiagramme, also auch das des Eem, als Totaldiagramme gezeichnet (d. h. Gesamtpollensumme $=100 \%)$. In den Interstadialdiagrammen sind die Anordnung der Kurven und der $\%$-Maßstab überall gleich, allen schwächeren Kurven wurde zusätzlich eine zehnfache Überhöhung aufgesetzt. Der Tiefenmaßstab ist ebenfalls in allen Diagrammen gleich, mit Ausnahme des obersten (Glinde), wo er auf das Doppelte gestreckt ist.

Für die noch laufende Bearbeitung der botanischen Makroreste steht ausreichend Material zur Verfügung. Deren Ergebnisse werden später vorgelegt, teilweise können sie jedoch schon in diesem Beitrag berücksichtigt werden. Ebenfalls in Arbeit befinden sich absolute Altersbestimmungen, die von Prof. МоOK, Groningen, durchgeführt werden.

\subsection{Das Eem-Interglazial und die Grenze Eem/Weichsel}

An der Basis der Profile von Oerel befindet sich ein gut ausgebildetes Eem, das in der Bohrung OE 61 bis cm 1684 in limnischer Fazies, darüber als Torf vorliegt (vgl. S. 18f und Taf. 1).

Die Vegetationsentwicklung im niedersächsischen Eem ist vor allem durch die Arbeiten von BEHRE (1962, hiernach auch die benutzte Gliederung) und SELLE (1962) bekannt; aus der näheren Umgebung von Oerel liegen bereits die Pollendiagramme von Köhlen (BENDA \& SCHNEEKLOTH 1969) und Osterwanna (BEHRE 1974) vor. In jüngster Zeit haben MENKE \& TYNNI (1984) die Ergebnisse der Eem-Untersuchungen aus Schleswig-Holstein mit denen anderer Autoren zusammengestellt und die großräumige Vegetationsentwicklung dieser Zeitperiode herausgearbeitet. Kennzeichnend für sie ist besonders die im Vergleich zum Postglazial große Gleichförmigkeit an den verschiedenen Lokalitäten.

In Oerel, OE 61, beginnt das Eem mit dem Abschnitt E IVa, der Hasel-EMW-Kiefernzeit, in der der Corylus-Gipfel mit 72,3\% des Gesamtpollens erfaßt ist. Noch weiter zurück, bis in den Abschnitt E III, reicht das Eem nur im Profil OE 22. Beim Vergleich mit anderen Eem-Diagrammen ist zu berücksichtigen, daß bei uns durch die Darstellung als Totaldiagramm alle Pollenwerte verändert sind: in einem 
Tab. 1: Gliederung des Spätpleistozäns von Oerel (Bohrung OE 61)

(Eem nach BeHRE 1962, WF I-IV nach MeNKE \& TYNNI 1984, WP I-IV neu)

$\mathrm{WF}=$ Weichsel-Frühglazial; $\mathrm{WP}=$ Weichsel-Pleniglazial

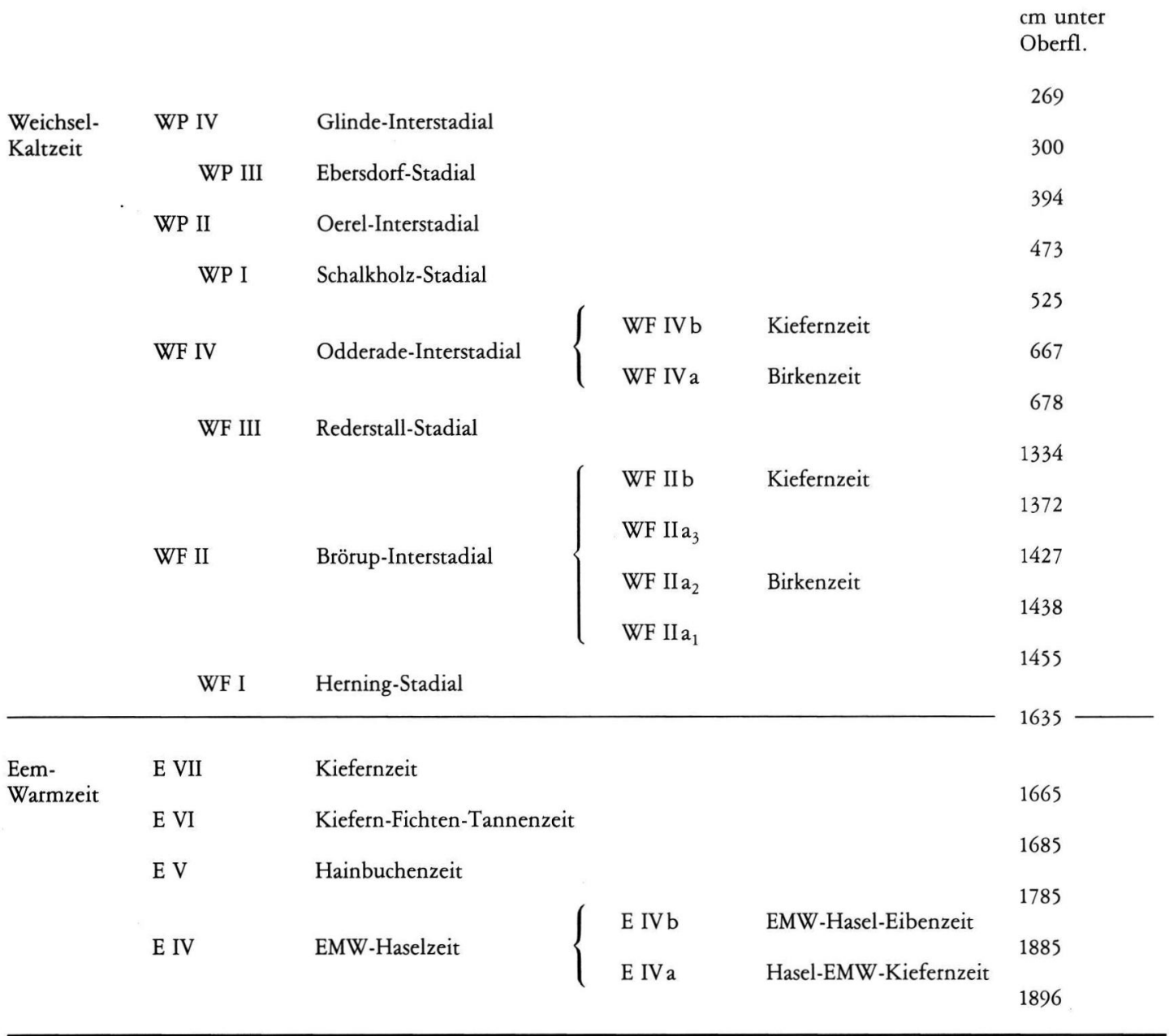

Baumpollendiagramm würde z. B. der Haselgipfel in Oerel $270,4 \%$ betragen. Abnehmende Quercus- und ansteigende Tilia- und Taxuswerte charakterisieren den Vegetationsablauf in diesem Abschnitt.

Der Abschnitt E IVb, die EMW-Hasel-Eiben-Zeit, umfaßt die hier gleichzeitige Kulmination der Tiliaund Taxuskurven und die langsame Ausbreitung von Carpinus und Picea, verbunden mit dem allmählichen Corylus-Rückgang.

In der Hainbuchenzeit, Abschnitt E V, dominiert Carpinus, hingegen bleibt Picea schwächer als in anderen Vorkommen. Wie auch andernorts in Seeablagerungen (vgl. hierzu BEHRE 1962) kommt es im jüngeren Teil von $\mathrm{E} V$ nicht zu einer Zunahme von Picea, die für (Bruchwald-) Torfe, z. B. in Köhlen, charakteristisch ist. Bemerkenswert ist der zweimalige Nachweis von Buxus gegen Ende von E V, der bisher westlich der Elbe noch fehlte.

Der Abschnitt E VI, die Kiefern-Fichten-Tannenzeit, wird von Anfang und Ende der Tannenkurve begrenzt. Abies erreicht im Elbe-Wesergebiet normalerweise Maxima von 4-6\%, die Arealgrenze lag nicht mehr weit entfernt und wurde bereits in Jütland nach Norden überschritten. Die ausgesprochen niedrigen Abies-Werte in Oerel (max. 1,3\%) sind wahrscheinlich durch eine kurze Schichtlücke verursacht, die am Fazieswechsel limnisch-telmatisch bei cm 1684 
liegt. Hierauf deutet auch der im Vergleich mit anderen Pollendiagrammen zu steile Abfall der Kurven von Carpinus, Alnus und Corylus hin. Im Abschnitt E VI erfolgt sehr schnell der Aufstieg von Pinus zur Dominanz und die erste Auflockerung der Wälder, erkennbar an der Zunahme des Kräuterpollens.

In der Kiefernzeit, Abschnitt E VII, geht die Öffnung der Wälder weiter, und es zeigt sich ein Phänomen, das für den Abschluß des Interglazials im Tiefland nördlich der Mittelgebirge charakteristisch ist: die allgemeine Versauerung der Böden. In Oerel macht sich diese Versauerung zuerst durch ungewöhnlich hohe Werte von Rumex (bis $17,2 \%$ ) bemerkbar, es folgt Calluna, und die Sphagnum-Kurve weist darauf hin, $\mathrm{daß}$ die Dystrophierung stellenweise bis zu einem Waldhochmoor führt.

Mit der starken Ausbreitung der Ericalesheiden - zunächst von Empetrum, dann von Calluna beherrscht - und dem entsprechenden Rückgang des Baumpollens folgt der Beginn der Weichselkaltzeit mit dem Herning-Stadial (WF I). Die Kriterien dieser Grenze wurden kürzlich von MENKE (in MENKE \& TYNNI 1984, S. 52 ff.) überarbeitet und festgelegt. Wichtig ist dabei nicht nur die allgemeine Zunahme der Ericales, sondern auch das Vorhandensein von Kräutern anderer Standorte, z. B. von Artemisia, deren geschlossene Kurve in Oerel bei cm 1640 einsetzt. Außerdem muß sichergestellt sein, daß hohe EricalesWerte nicht allein auf Hochmoorbedingungen zurückzuführen sind. Das trifft in Oerel für die obersten Proben zu.

\subsection{Das Brörup-Interstadial}

Das zwischen dem Eem und dem Brörup liegende Herning-Stadial (WF I nach MENKE \& TYNNI 1984) wurde über dem Eem zunächst noch in Form von Torf erfaßt (cm 1635-1609). Darüber liegt ein niveofluviatil transportierter Mittelsand, der bei $\mathrm{cm} 1527$ von einer olivfarbenen, zunächst stark schluffigen Mudde abgelöst wird, auf die ab cm 1437 ein Torf folgt (Taf. 2).

In den limnischen Ablagerungen ab cm 1527 ist zunächst noch das Herning-Stadial, WF I, reflektiert.
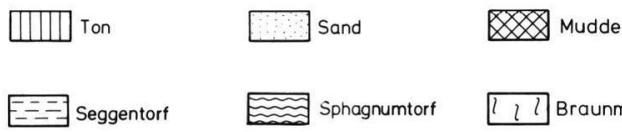

E-ニニ- Seggentorf

l l l Braunmoostorf

Eolzreste

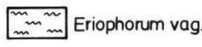

Abb. 11: Legende zur Stratigraphie der Pollendiagramme.
Mit dem eingewaschenen Schluff erscheint auch viel Sekundärpollen wie Alnus, Picea und thermophile Laubhölzer. Auch Sphagnumsporen und Calluna dürften in diesem Bereich überwiegend umgelagert sein. Von etwa cm 1495 geht die Einschwemmung zurück; die Zunahme des Nichtbaumpollens im Diagramm ist nur scheinbar, da der Anteil des umgelagerten Baumpollens abnimmt. Die Vegetationsdecke schließt sich, und die spät-stadiale Vegetation wird erkennbar: viele Gramineae und Heliophyten wie Artemisia, Thalictrum, Plantago, Helianthemum usw. Betula nana und Juniperus waren als Sträucher vorhanden.

Bemerkenswert sind einzelne Pollenkörner unter den Gramineen, die nach der Größe dem Getreidetyp zugeordnet werden müßten, die genauere morphologische Untersuchung weist sie jedoch bislang unbekannten Wildgräsern zu. Sie haben eine Größe von meist um $50 \mu$, maximal $58 \mu$, und ihr Auftreten beschränkt sich auf die stadialen Mudden vor dem Brörup (17 Pollenkörner) und vor dem Odderade (ebenfalls $17 \mathrm{PK})$. Vermutlich die gleiche Art ist gelegentlich bereits in anderen Interstadialen gefunden und als „Cerealia-Typ” vermerkt worden, so von V. D. MEER et al. 1984 in Eerbeek IV (Flachwassersediment, wahrscheinlich Hengelo) und von PESCHKE 1983 in Herrnhausen (Schieferkohle, nach PESCHKE älteres Interstadial).

Ein scharfer Wechsel erfolgt bei cm 1455 mit dem Steilanstieg von Betula, nach Ausweis der Makroreste ist es die Ausbreitung der baumförmigen Birken. Diese starke Klimaänderung, die zur Bewaldung führt, wird als Grenze zum Brörup, WF II, genommen. Der Begriff Brörup wird hier im Sinne von ANDERSEN (1961) benutzt, wie er am locus typicus entwickelt wurde (s. u.). Unterteilt wird das Brörup in die Grundeinheiten Birkenzeit, WF IIa, und Kiefernzeit, WF IIb. Durch die Bewaldung sinkt der Grundwasserstand, und bei $\mathrm{cm} 1432$ wird die Mudde von Torf abgelöst. Innerhalb der Birkenzeit erfolgt ein wahrscheinlich klimatisch bedingter Rückschlag der Vegetation (WF $\mathrm{IIa}_{2}$ ), durch den die Birkenzeit in WF $\mathrm{IIa}_{1}-\mathrm{a}_{2}-\mathrm{a}_{3}$ dreigeteilt wird. Dieser Rückschlag ist auch im benachbarten Pollendiagramm von Osterwanna (BEHRE 1974) zwischen den Proben 32 und 45 erkennbar. Wie später ausgeführt wird, entspricht WF IIa 1 vermutlich dem Amersfoort, und ab WF IIa 3 beginnt das Brörup im Sinne von ZAGWIJN (1961). Die Zunahme des Nichtbaumpollens im Abschnitt WF IIa ${ }_{3}$ ab cm 1415 ist wahrscheinlich nicht auf klimatische Ursachen zurückzuführen, denn in diesem Zeitraum erfolgt gleichzeitig die Einwanderung neuer Baumarten, wie Picea und Larix, und auch thermophile Laubhölzer, vor allem Quercus und Corylus, haben durchlaufende Kurven. Das Klima ist demnach 
zumindest stabil geblieben. Die Ausbreitung von Picea (und Larix) erfolgt auch in den Brörup-Diagrammen von Amersfoort (ZAGWIJN 1961), Rederstall (MENKE \& TYNNI 1984) und Osterwanna immer erst deutlich nach dem Klimarückschlag. In der Regel hat Larix einen Vorsprung, in Oerel breiten sich dagegen beide Arten etwa gleichzeitig aus.

Das regelmäßige Auftreten der thermophilen Laubholzarten kann im Torf nicht auf Einschwemmung beruhen, sondern die Pollenkörner müssen fernverweht sein. Ganz ähnliche Nachweise liegen aus dem benachbarten Osterwanna und den schleswig-holsteinischen Brörupvorkommen vor, zum Teil aus reinen Hochmoortorfen. Wenn in den Pollenspektren dieses dicht bewaldeten Interstadials oftmals eine Summe von über $1 \%$ thermophiler Arten auftritt, so können deren Bestände nicht allzuweit entfernt gewesen sein. Bei BEHRE 1974 (S. 17 ff.) sind die Klimaverhältnisse im hiesigen Brörup ausführlich beschrieben; durch den Vergleich mit postglazialen Pollendiagrammen aus Nordeuropa konnte abgeschätzt werden, daß die thermophilen Holzarten ihre nördlichen Arealgrenzen während des Brörup etwa 4-6 Breitengrade südlich von Osterwanna (und damit auch von Oerel) hatten, also etwa zwischen Main-Linie und Alpennordrand. Angenommen wird dabei ein etwa dem heutigen gleicher Nord-Süd-Klimagradient. Die 1975 erschienene Bearbeitung von Grande Pile durch WoILLARD bestätigte das vollständig. Die (gelegentlich angezweifelte) Synchronisierung des nordmitteleuropäischen Brörup mit dem St. Germain I-Interstadial südwestlich der Vogesen entspricht damit auch der damaligen, nach S verschobenen, Zonierung der Wälder. Für das Alpenvorland zeigten GRÜGER (1979: 22) und WELTEN $(1981,1982)$ Entsprechendes, wenn man die dortige Höhenlage berücksichtigt. Ein Vorkommen der Eichenmischwaldarten sowie von Carpinus oder Corylus in Nordwestdeutschland und Dänemark ist auszuschließen. Die Klimagrenzen im Brörup müssen sehr lange stabil gewesen sein, aber das Klima in Norddeutschland hat thermophile Laubhölzer nicht zugelassen, denn bei der langen Dauer des Interstadials wäre für deren Einwanderung Zeit genug vorhanden gewesen.

Die inzwischen mehrfach bestätigte Gleichsetzung des Brörup mit dem St. Germain I und dem 1. Interstadial am Samerberg hat zur Konsequenz, daß mehrere bisher ins Brörup gestellte Interstadiale in Süddeutschland umgeordnet werden müssen (FRENZEL 1980 , S. 53), und macht als weitere Folge eine Umzeichnung der bisher vorgelegten Klimakarten dieses Zeitraumes nötig.

Den Abschluß des Brörup-Interstadials bildet die Kiefernzeit, WF IIb. Sie ist im Profil OE 61 nicht vollständig, der oberste Teil fehlt hier. In der benach- barten Bohrung OE 46 wurde jedoch ein vollständiges Brörup erfaßt und deshalb zur Ergänzung untersucht. Dort umfaßt der Abschnitt IIb etwa $1 \mathrm{~m}$, nach oben hin gehen Larix und Picea wieder zurück, während die Ericales (vor allem Calluna) über $30 \%$ des Gesamtpollens erreichen. Auch das Pollendiagramm des unteren (Brörup-) Interstadials bei SELLE \& SCHNEEKLOTH 1965 ist nahezu vollständig und erfaßt fast die ganze Kiefernzeit.

\subsection{Das Odderade-Interstadial}

Auf den Torf des Brörup-Interstadials folgen bei $\mathrm{OE}$ 61 ohne Übergang fast $6 \mathrm{~m}$ mächtige niveofluviatile Mittel- und Grobsande, z. T. mit Feinkies-Anteilen, abgelagert im Rederstall-Stadial. Die gleichalte Schicht bei OE 46 ist dagegen als mittelsandiger Feinsand mit sehr geringen Grobsand-Anteilen ausgebildet. Dieser Körnungsunterschied ist wahrscheinlich der Grund für das Fehlen des obersten Teiles von WF IIb in OE 61: hier starke Strömung der Schneeschmelzwässer mit partieller Erosion der Btörupschicht, dort schonende Ablagerungsbedingungen.

$\mathrm{Ab}$ cm 756 setzt eine olivgrüne stark feinsandige Schluffmudde ein, die ebenfalls noch in das Rederstall-Stadial gehört. Sie führt etwas Sekundärpollen (z. B. Alnus, Picea), reflektiert aber im übrigen gut die Vegetation des ausgehenden Stadials (Taf. 2). Dabei sind einige typische Heliophyten, wie Helianthemum, Armeria und Gypsophila, erheblich häufiger als in der entsprechenden Phase vor dem Brörup. In ähnlicher Weise wie dort breitete sich auch Juniperus aus. Die Wasserpflanzen waren gut entwickelt und spiegeln einen interessanten Wechsel des Trophiegrades wider: Zunächst herrschen eu- bis mesotrophe Verhältnisse, gekennzeichnet durch Pollen von Myriophyllum spicatum/verticillatum, dann erfolgt bei cm 715 ein Umschlag zu oligotrophen Verhältnissen, erkennbar an dem sehr häufigen Vorkommen von Myriophyllum alterniflorum. Offenbar waren während der vorangegangenen stadialen Bodenbewegungen neue Nährstoffe aufgeschlossen worden, die aber bald wieder erschöpft waren. Ganz ähnliche Erscheinungen gibt es auch im frühen Brörup in Rederstall (MENKE 1980: 113) und im Weichsel-Spätglazial (z. B. b. BEHRE 1966 sogar mit den gleichen Arten). Gegen Ende des Rederstall-Stadials kommt es zur Verlandung: Typha/Sparganium und hohe Gramineenwerte zeigen den Uferbereich, und bei $\mathrm{cm} 668$ setzt der Torf ein.

Noch in der Muddephase breiten sich mit großer Geschwindigkeit die baumförmigen Birken aus, und es kommt zur Bewaldung. Mit diesem scharfen Vegetations- und Klimawechsel beginnt das Odderade-Interstadial, WF IV. Wie zu Beginn des Brörup 
bewirkt auch jetzt der Beginn der Bewaldung, vermutlich durch die erheblich verstärkte Transpiration und besseres Festhalten der Niederschläge, eine Absenkung des Grundwasserspiegels, so daß der See erblindet und bei $\mathrm{cm} 668$ das Torfwachstum beginnt.

Vergleichbare bewaldete Interstadiale über dem Brörup sind im Tiefland nördlich der Mittelgebirge bisher außer Oerel nur wenige publiziert worden: wichtig sind davon die Typuslokalität Odderade (AVERDIECK 1967), das ihm benachbarte Rederstall (MENKE \& TYNNI 1984) und Kittlitz in der Niederlausitz (ERD 1973). In allen diesen Vorkommen kann das Odderade in eine Birkenzeit, WF IVa, und eine Kiefernzeit, WF IVb, gegliedert werden. Im Unterschied zum Brörup ist die Birkenzeit im Odderade allerdings immer sehr kurz; entweder ging die Klimabesserung schneller oder — wahrscheinlicher — war das Pinusareal nicht so weit zurückgedrängt wie vor dem Brörup, so daß die Kiefer schneller wieder einwandern konnte.

Bei der ähnlichen Vegetationsentwicklung im Brörup und Odderade ist sorgfältig auf die Unterschiede zwischen beiden zu achten, die sich in allen bisher bekannten nordmitteleuropäischen Vorkommen abzeichnen: Neben der verschieden langen Birkenzeit ist es vor allem die Ausbreitung von Picea und Larix, die im Brörup in der Birkenzeit, im Odderade jedoch erst in der Kiefernzeit erfolgt. Beide Bäume erreichen im Brörup erheblich höhere Pollenwerte als im Odderade, außerdem ist die Ausbreitungsfolge im Brörup meist Larix - Picea (nur in Oerel etwa gleichzeitig), im Odderade dagegen Picea - Larix. Einen weiteren Unterschied liefert in Nordwestdeutschland die Alnus-Kurve; sie erreicht im Brörup meist 5-10\%, bleibt dagegen im Odderade unter $1 \%$. Bei den geringen Werten des EMW sowie von Carpinus und Corylus gibt es zumindest in Oerel für diese Gehölze keine deutlichen Unterschiede zwischen den beiden Interstadialen. Das bedeutet auch, daß die Arealgrenzen der thermophilen Arten im Brörup und Odderade sehr ähnlich gewesen sein müssen. Bestätigt wird dieser Befund im südlichen Mitteleuropa, wo die thermophilen Bäume in beiden Interstadialen etwa gleich stark vertreten sind (Grande Pile, WOILlard 1975; Sulzberg, WeLTEN 1981; Les Echets, DE BEAULIEU \& REILLE 1984).

In Oerel bleibt die Entwicklung während der Kiefernzeit, WF IVb, recht gleichförmig. Die relativ schwache Ausbreitung von Picea und Larix verändert den Wald nur wenig. Am Standort des Profils wird ein Zwischenmoor schnell von einem zeitweise sehr Sphagnum-reichen Waldhochmoor abgelöst. Gegen Ende des Interstadials nehmen auf dem Hochmoor die Ericales (Empetrum, Calluna) sowie Rubus chamaemorus stark zu. Wie die übrigen Nichtbaumpollenkurven zeigen, wird das folgende Stadial im Pollendiagramm nicht mehr erfaßt.

\subsection{Das Oerel-Interstadial}

Auf das Odderade-Interstadial folgt von $\mathrm{cm} 525$ bis cm 473 hellgrauer Feinsand, der zunächst stark, später schwach mittelsandig und erkennbar geschichtet ist und im Schalkholz-Stadial (Bezeichnung nach MENKE \& TYNNI 1984) abgelagert wurde. Humose Einlagerungen fehlen, so daß angenommen werden kann, daß das Stadial kalt und \pm vegetationslos war.

Der sterile Sand wird übergangslos von einem Torf abgelöst, der zunächst mäßig zersetzt ist und überwiegend aus Braunmoosen und Carex gebildet wurde, Reste von Betula nana sind ebenfalls beteiligt. Nach oben nimmt der Zersetzungsgrad dieses Braunmoostorfs $\mathrm{zu}$, bis ab cm 433 ein schwach zersetzter Torf folgt. Dieser wird von Braunmoosen und nach oben zunehmend von Sphagnum gebildet, ab cm 415 ist es ein fast reiner Sphagnumtorf, z. T. mit viel Eriophorum vaginatum. Sehr hohe Werte von Sphagnumsporen kennzeichnen diese Phase auch im Pollendiagramm. Die Entwicklung ging hier also offensichtlich von einem zunächst mesotrophen grundwassernahen Standort bis zum reinen Hochmoor außerhalb des Mineralbodenwasserbereichs. Das Niederschlagsnetto muß demnach zur Bildung ombrotropher Vegetation ausgereicht haben.

Die Torfe dieses „Oerel” genannten Interstadials sind in mehreren Bohrungen angetroffen und pollenanalytisch korreliert worden, das Vorkommen erstreckt sich über eine größere zusammenhängende Fläche (vgl. Abb. 3). Es handelt sich um die gleiche Torfschicht, die bereits SELLE in einer Stärke von $32 \mathrm{~cm}$ über den beiden großen Interstadialen erbohrt hat und die ohne ausdrückliche Benennung als „,oberes Interstadial von Oerel" bekannt wurde (SELLE \& SCHNEEKLOTH 1965: 110 ff.). Die SELLEsche Bohrung liegt an der Stelle unserer Bohrung OE 14 (vgl. Abb. 3).

Der Vegetationsablauf im Oerel ist gekennzeichnet durch hohe Nichtbaumpollenwerte, die im Mittel $81,3 \%$ betragen. Bäume sind während dieses Interstadials hier nicht vorgekommen; die bis $23,6 \%$ betragenden Betula-Werte müssen von Betula nana stammen. Die sehr genau durchgeführten MakrorestAnalysen brachten keinerlei Reste von Baumbirken, stattdessen aber aus dem ganzen Interstadial zahlreiche Blattreste sowie Früchte und Fruchtschuppen von der Zwergbirke, Betula nana. Neben der Zwergbirke sind an Sträuchern im ersten Abschnitt vorwiegend Weiden (Salix) und im zweiten Abschnitt 
Wacholder (Juniperus) am Ort gewachsen. Pinus, Alnus und die einzelnen Pollenkörner anderer Bäume sind fernverweht.

Die nördliche Baumgrenze ist weit entfernt gewesen. Als Vergleich kann man den rezenten Pollenniederschlag arktischer und subarktischer Gebiete heranziehen, z. B. die immer noch wichtige Arbeit von AARIO (1940) aus Petsamo-Lappland oder die neueren Untersuchungen von LICHTI-FEDEROVICH \& RITCHIE (1968) und LAMB (1984) aus Kanada. Danach erreicht der Baumpollen (einschl. Betula) in Oberflächenspektren aus der Tundra noch weit nördlich der Baumgrenze Werte um und über $50 \%$, wobei der fernverwehte Nadelholzpollen eine besondere Rolle spielt. Die Betulawerte, die überwiegend von den am Ort vorhandenen Beständen der Zwergbirken ( $B$. nana, bzw. in Kanada $B$. glandulosa) stammen, erreichen im Mittel 20-30\%, was etwa den Verhältnissen im Oerel entspricht.

Kennzeichnend für das Oerel-Interstadial ist die starke Ausbreitung der Zwergsträucher Calluna und vor allem Empetrum. Zwar können sich im Empetrum-Pollentyp auch andere Ericales-Arten, vor allem Ledum, verstecken, doch dürfte es sich im Pollendiagramm des Oerel wohl tatsächlich nur um Empetrum handeln, denn die Makroreste lieferten von cm 420 an nach oben regelmäßig Samen von Empetrum nigrum, an weiteren Ericales dagegen nur einzelne Samen von Arctostaphylos uva-ursi, deren Pollen in den "übrigen Ericaceae" enthalten ist. Diese Nachweise zeigen, daß Empetrum auch lokal auf dem Hochmoor wuchs und seine Pollenwerte dadurch erhöht sind. Empetrum ist auch in den Pollenanalysen des Oerel aus anderen OE-Profilen regelmäßig, aber meist schwächer vertreten. Besonders große Häufigkeit, verbunden mit nennenswerten Mengen an Samen, zeigt das etwa $60 \mathrm{~m}$ von OE 61 entfernte alte Profil des „oberen Interstadials” (vgl. SCHNEEKLOTH 1966). Der dort ebenfalls angegebene Pollen von Hippophae konnte von uns allerdings nicht wiedergefunden werden; möglicherweise handelt es sich dabei um Rumex/Oxyria-Pollen.

Insgesamt zeigt das Pollendiagramm für das OerelInterstadial eine baumlose offene Strauchtundra. Kräuter sind in großer Artenzahl vorhanden, doch herrschten, abgesehen von der lokalen Hochmoorbildung, keine besonders feuchten Bedingungen, sonst wären vor allem die Cyperaceenwerte wesentlich höher. Bemerkenswert sind die wohl erstmaligen Pollenfunde von Linum anglicum ( = L. perenne ssp. anglicum), deren porate Pollenkörner nach den Merkmalen von PUNT \& DEN BREEJEN (1981) gut zu bestimmen sind. Diese Art ist nach MeuSEL et al. (1978) ein spätglaziales Steppenrelikt, erreicht aber mit mehreren rezenten Fundplätzen die nördlichen Tundrenzonen.

\subsection{Das Glinde-Interstadial}

Auf den Torf des Oerel-Interstadials folgt erneut übergangslos von $\mathrm{cm} 394$ bis $\mathrm{cm} 300$ ein reiner gelbgrauer, schwach mittelsandiger Feinsand, abgelagert im Ebersdorf-Stadial (vgl. s. 21). Dieses muß wiederum ein kaltes Stadial gewesen sein, denn es fehlen jegliche autochthonen organischen Reste, lediglich sehr geringe Mengen von eingeschwemmtem aufgearbeitetem Pflanzenhäcksel wurden angetroffen.

Der Beginn des darauffolgenden Glinde-Interstadials (vgl. S. 22) unterscheidet sich pollenanalytisch in vielfacher Weise vom Ende des Oerel, so daß eine längere Zwischenzeit zu postulieren ist.

Diese jüngste interstadiale Folge setzt ein mit einem Seggentorf, der zunächst unter nassen, fast limnischen Bedingungen abgelagert worden ist und viel Carex, dazu Eleocharis palustris-Früchtchen, Pollen von Lythrum, Batrachium usw. enthält. Bei cm 279 erfolgt eine noch stärkere Vernässung mit einem Übergang zur Feindetritus-Mudde, und ab cm 273 liegt nur noch ein feinsandiger Schluff mit humosen Lagen vor. Von $269-260 \mathrm{~cm}$ folgt ein sandiger Kies und darauf weitgehend steriler weichselzeitlicher Sand.

Auch der Torf des Glinde-Interstadials ist flächig ausgebildet. Er wurde in den Profilschnitten 1 und 5 sowie in zusätzlichen Sondierungen angetroffen. Seine ungefähre Ausdehnung ist in Abb. 3 (rechts oben) umgrenzt. Da die organische Lage des Glinde in OE 61 nur $30 \mathrm{~cm}$ mächtig ist, wurde sie im Pollendiagramm (Taf. 2) gegenüber den tieferliegenden Schichten auf das Doppelte gestreckt, was beim Vergleich zu beachten ist.

Der Vegetationsablauf zeigt, daß auch das GlindeInterstadial baumlos und durch eine Strauchtundra gekennzeichnet war (durchschnittlicher NBP-Wert $=78,2 \%)$. Entsprechend den lokalen Ablagerungsverhältnissen sind Arten aus dem feuchten Bereich stark vertreten, doch die Cyperaceae etwa erreichen nie $60 \%$, und Pollendiagramm sowie Makroreste reflektieren auch trockenere Gebiete der Umgebung. Der Anteil der Sträucher und Zwergsträucher war im Glinde-Interstadial deutlich schwächer als im Oerel: wichtig ist vor allem, daß Juniperus fast völlig fehlt und die Ericales erheblich zurücktreten. Die Makroreste zeigen jedoch, daß Empetrum nigrum fast während des ganzen Interstadials am Orte vorhanden war. Salix erreicht als Pollen im unteren Teil 9,1\% und ist auch als Holz vertreten. Die Werte von Betula sind etwas höher als im vorangegangenen Interstadial; auch aus dem Glinde gibt es Makroreste von Betula nana, jedoch nicht von Baumbirken. 


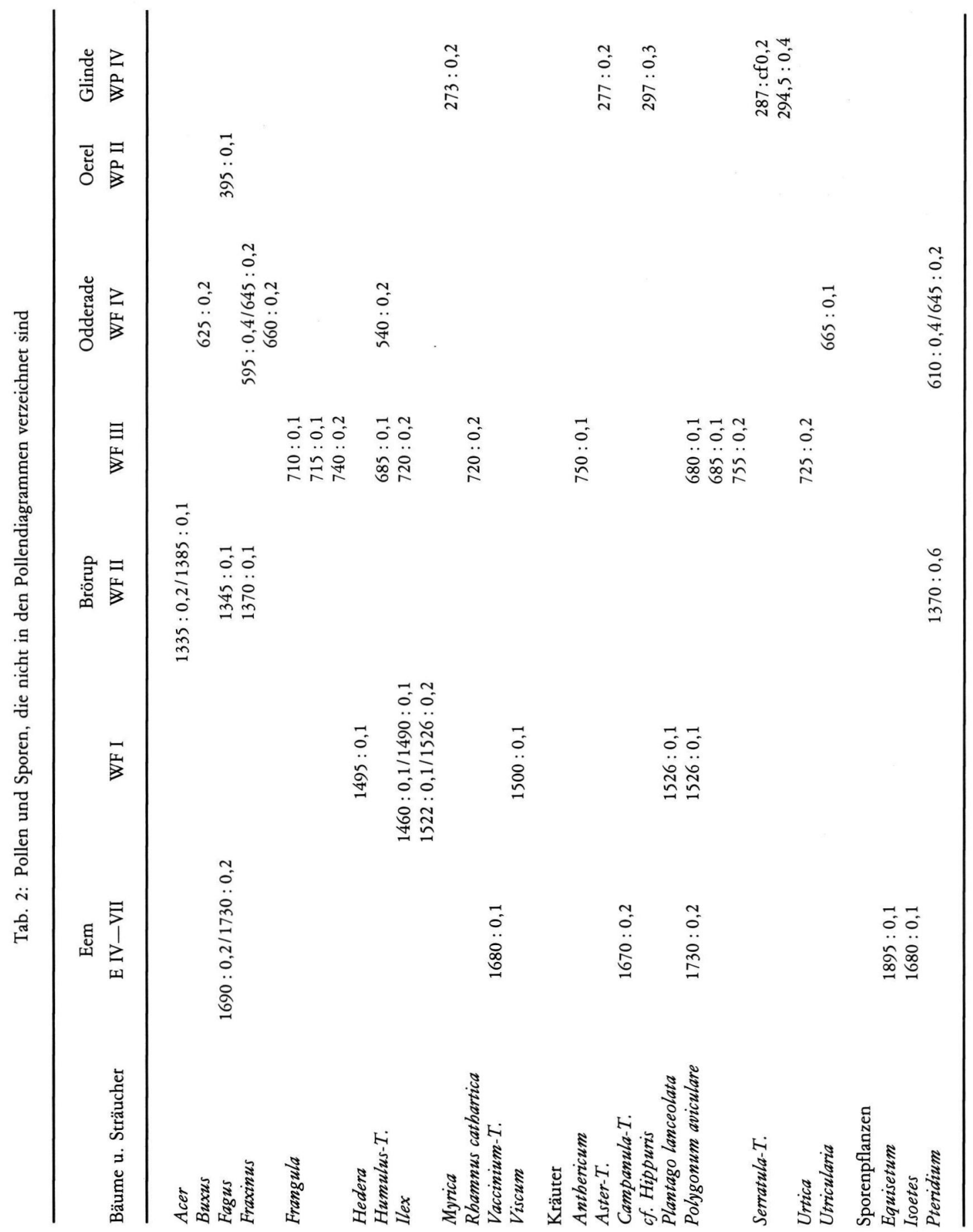


Insgesamt ist aus der Pollenflora erkennbar, daß die Vegetation während des Glinde offener war als im Oerel: charakteristische Heliophyten, wie Helianthemum, Armeria und die Campanulaceae, treten im Glinde wesentlich häufiger auf; Selaginella selaginoides fehlt im Oerel, ist aber im Glinde nicht nur mit Mikrosporen, sondern mehrfach sogar mit Megasporen vorhanden. Plantagopollen ist mit einem Maximum von $7,7 \%$ im Glinde häufig. Die weichselzeitliche Plantago-Art ist nach wie vor unbekannt; die Pollenkörner liegen morphologisch meist zwischen dem $\mathrm{Pl}$. maritima-Typ und dem $\mathrm{Pl}$. maior/mediaTyp, wobei die Ähnlichkeit mit dem letzteren größer ist als mit Pl. maritima. Nach dem Klima zu urteilen, ist hier wohl vor allem mit Plantago alpina zu rechnen, der morphologisch auch hierhin gehört.

Bemerkenswert sind die sehr geringen Pinus-Werte im Glinde, die im Durchschnitt nur 0,95\% betragen (gegenüber 2,9\% im Oerel). Dies ist ein sicherer Hinweis darauf, daß die nächsten Kiefernbestände sehr weit entfernt waren und - nachdem sie während des zwischen Oerel und Glinde liegenden EbersdorfStadials weit zurückgedrängt worden waren - im Verlauf des Glinde nicht wieder so weit vordringen konnten wie vorher.

Festzuhalten ist, daß die Interstadiale Oerel und Glinde sich an der Typuslokalität pollenanalytisch deutlich unterscheiden lassen. Bei den Holzgewächsen sind die entscheidenden Taxa Juniperus, Pinus und die Ericales, bei den Kräutern vor allem Filipendula, Plantago und Selaginella. Hinzu kommt die unterschiedliche Ausbildung der Torfe.

\subsection{Zur zeitlichen Einordnung der Interstadiale}

Oerel und Glinde nach dem Vegetationsbild

Über die Anzahl und die Vegetation der WeichselInterstadiale sind wir nach wie vor unzureichend unterrichtet. Als sicher kann angesehen werden, daß es im nördlichen Mitteleuropa außer den großen Interstadialen Brörup s. 1. und Odderade keine weiteren bewaldeten Weichsel-Interstadiale gab. Unbewaldete jüngere Interstadiale sind vor allem aus den Niederlanden mehrfach beschrieben worden (VAN DER HAMMEN et al. 1967; VAN DER HAMMEN 1971; ZAGWIJN 1974; KOLSTRUP \& WiJMSTRA 1977 usw.). Die meisten dieser Vorkommen lassen sich in die Interstadiale Moershoofd, Hengelo und Denekamp einordnen, wobei vor allem die ${ }^{14} \mathrm{C}$-Daten entscheidend für die Eingruppierung sind. Daneben gibt es auch mehr oder weniger deutliche Unterschiede in der Vegetation, die für das norddeutsch-niederländische Tiefland im Hengelo und im Denekamp meist eine Strauchtundra annehmen lassen, während das Moershoofd als offener, arm an Sträuchern und im ganzen kühler gilt. Sowohl die Pollenanalysen als auch die stark streuenden ${ }^{14} \mathrm{C}$-Daten lassen vermuten, daß der MoershoofdKomplex in mehrere voneinander getrennte Interstadiale aufgegliedert werden muß.

Nach den lang durchlaufenden Weichsel-Profilen von Grande Pile (WOILLARD 1975, 1978), Sulzberg (Welten 1982), Les Echets (DE BEAULIEU \& ReILlE 1984) und Tenagi Philippon (WIJMSTRA 1969) und deren wahrscheinlicher Übereinstimmung mit der Sauerstoff-Isotopen-Stratigraphie im Nordatlantik (SHACKLETON 1977, vgl. auch WOILLARD 1978) ist zu erwarten, daß es außer den bisher bekannten noch weitere Interstadiale im Weichsel-Pleniglazial gab, die in Mitteleuropa unbewaldet waren. Für die oberen Interstadiale in Oerel sind ${ }^{14} \mathrm{C}$-Datierungen in Arbeit, doch sollen hier zunächst mögliche Parallelisierungen mit bekannten Interstadialen anhand der Vegetation geprüft werden.

Ein Vergleich des Oerel-Interstadials mit den bekannten Weichsel-pleniglazialen Interstadialvorkommen der Niederlande läßt sehr schnell erkennen, daß es für das Oerel dort keine Parallele gibt. Die MoershoofdDiagramme, z. B. Denekamp/Bohrung 20a (VAN DER HAMMEN 1971), Hengelo/Bohrung 1 u. 2 a (ZAGWIJN 1974), Voorthuizen und Eerbeek (KolsTRUP \& WIJMSTRA 1977, VAN DER MEER et al. 1984) sowie Duckenburg (TEUNISSEN et al. 1974) zeigen eine wesentlich offenere Vegetation mit mehr Heliophyten, wie Artemisia, Helianthemum usw. Vor allem unterscheiden sie sich vom Oerel dadurch, daß sie kaum oder keine Ericales aufweisen. Auch Juniperus ist nur spärlich nachgewiesen.

Die Interstadiale Hengelo und Denekamp, z. B. Ruigekluft und Hengelo (ZAGWIJN 1974), Bussloo und Laarhuis (KOLSTRUP \& WIJMSTRA 1977) kommen ebenfalls für eine Korrelation mit dem Oerel nicht in Betracht: Oerel hat noch mehr Sträucher als dort vorkommen (vor allem viel mehr Ericales, auch Betula nana) und entsprechend wesentlich weniger Kräuter (Cyperaceae, Artemisia, Helianthemum usw.). Klimageschichtlich wichtig ist auch, daß es im Oerel nicht nur zur Torf-, sondern sogar zur Hochmoorbildung kam, die in den Niederlanden nicht mehr stattfand; dort entstanden in den genannten Interstadialen überhaupt nur selten reine Torfe.

Das in Oerel zuoberst liegende Glinde-Interstadial ist im Vegetationscharakter und damit im Klima den niederländischen pleniglazialen Interstadialen bereits ähnlicher als das vorangehende Oerel. Es herrschte eine offene Strauchtundra, die klimatisch vermutlich zwischen den Verhältnissen im Moershoofd und im Hengelo/Denekamp lag. Von Bedeutung sind die im 
Glinde höheren Werte von Betula (nana) und der Ericales sowie die im Vergleich zu den Niederlanden äußerst niedrigen Werte von Pinus (im Mittel unter $1 \%)$, die auf eine sehr große Entfernung der Kiefernbestände schließen lassen. Zu einer Hochmoorbildung ist es im Glinde nicht mehr gekommen.

Nach dem Vegetationsbild der beiden oberen Interstadiale von Oerel kann angenommen werden, daß sie vor den niederländischen pleniglazialen Interstadialen liegen; vielleicht reicht das Glinde noch in den frühen Bereich des Moershoofd-Komplexes. Wichtig für diese Annahme ist das im Oerel sehr starke und im Glinde immer noch häufige Auftreten der Ericales. Sie weisen auf den seit dem späten Eem sauren Charakter der Böden, der sich erst im Laufe des Pleniglazials durch die frostbedingte Bodenerneuerung ändert. Obwohl die jeweilige lokale Situation der Vorkommen eine Rolle spielt, zeigt die allgemeine Tendenz der Ericales-Häufigkeit im Verlauf der Weichsel-Kaltzeit nach unten. Die hohen Ericales-Werte am Ende von Eem, Brörup und Odderade werden nur noch im Oerel erreicht. Im gleichen Maße, wie im Laufe der Weichselstadiale durch dauerfrostbedingte Umlagerungen, wie z. B. Kryoturbation, Bodenfließen und niveofluviatilen Transport sowie durch äolische Ablagerungen die Böden nährstoffreicher werden, nehmen die Ericales ab. Bezeichnend dafür ist, daß sie auch während des Spätglazials in der Älteren Dryaszeit noch sehr schwach vertreten sind und sich erst in der Jüngeren Dryaszeit nach der im Alleröd wieder einsetzenden Bodenauswaschung erneut ausbreiten. Somit kann man unter vergleichbaren Bodenbedingungen wie im norddeutsch-niederländischen Tiefland die Ericales-Häufigkeit als Anhalt für eine relative Abfolge der Weichsel-Interstadiale benutzen.

In der Hohlform von Oerel sind zwischen dem Eem und dem Glinde keine Schichtlücken erkennbar. Die Ablagerungen erfolgten offenbar kontinuierlich, so daß eine Vollständigkeit der Interstadiale angenommen werden kann, bis nach dem Glinde die Hohlform aufgefüllt wurde. Ähnlich ungestörte Verhältnisse hat es nach MENKE $(1976,1980)$ in Keller und Schalkholz, beides in Schleswig-Holstein, gegeben. Dort ist oberhalb des Odderade ein Boden entwickelt, den MENKE als „Keller-Interstadial” beschrieben hat. Über die Vegetation im „Keller” ist nichts bekannt, doch ist dieser Boden wesentlich schwächer entwiçkelt als die darunterliegenden Böden von Brörup und Odderade, weswegen er zu einem relativ kurzen unbewaldeten Interstadial gehören könnte. Die ungestörte Situation spricht dabei für eine Korrelation mit dem Oerel.

Im überregionalen Vergleich muß vor allem eine Korrelation mit dem Standardprofil von Grande Pile südwestlich der Vogesen (WOILLARD 1975, 1978) versucht werden. Eine ungestörte Entwicklung hier und dort vorausgesetzt, wäre eine Synchronisation des Oerel mit dem dortigen Ognon-Interstadial möglich. Von den drei bei WOILLARD genannten Ognon-Interstadialen ist wohl nur das Ognon II als richtiges Interstadial zu werten; es wird von GRÜGER (1979a u. b) mit dem 3. Interstadial am Samerberg, von WeLTEN (1981, 1982) mit seinem Dürnten-Interstadial synchronisiert. Entsprechend der südlichen Lage aller dieser Vorkommen ist dieses Interstadial an allen genannten Lokalitäten bewaldet: am Samerberg und in Dürnten und Sulzberg in Form von Nadelwäldern, in Grande Pile auch mit Laubwaldanteilen. Ob eine Korrelation mit dem 3. Interstadial von Les Echets bei Lyon (DE BEAULIEU \& REILLE 1984) möglich ist, erscheint sehr fraglich.

In den langen Weichselprofilen von Grande Pile und Les Echets gibt es ebenso wie in Tenagi Philippon in Griechenland (WIJMSTRA 1969) mehrere weitere Erwärmungsphasen; eine Synchronisierung einer dieser Phasen mit dem Glinde ist nicht möglich, solange keine absoluten Altersangaben vorliegen.

\section{Diskussion}

Die Senke von Oerel liefert die Möglichkeit, eine kontinuierliche Abfolge des Vegetations- und damit des Klimaablaufs vom Eem bis in das Weichsel-Pleniglazial zu untersuchen, die kaum durch Erosionsvorgänge gestört wurde. Die Profile im tiefsten Teil der Hohlform, dargestellt an der Bohrung OE 61, ergaben vier organogene Interstadialbildungen, deutlich durch minerogene Stadial-Sedimente voneinander getrennt: Zwei bewaldete und darauf folgend zwei in unserem Raum unbewaldete Interstadiale.

Wie bereits auf S. $25 \mathrm{f}$ dargelegt, entsprechen die beiden bewaldeten Interstadiale von Oerel dem dänischen Brörup und dem Odderade. Man kann bei den geschilderten Ablagerungsverhältnissen mit Sicherheit davon ausgehen, daß es hier kein weiteres bewaldetes Interstadial gegeben hat, denn dieses hätte sich deutlich im Sediment abzeichnen müssen. Zwischen Eem und Brörup sowie vor und nach dem Odderade gibt es in den weitgehend sterilen Sanden keinerlei Hinweise auf zusätzliche Thermomere.

Die in den vergangenen Jahren sehr lebhafte Diskussion um die Korrelierung dieser nordwestdeutschdänischen Folge mit den von ZAGWIJN (1961) in Amersfoort benannten „Amersfoort" und „Brörup"Interstadialen läuft letztlich auf nur eine mögliche Lösung hinaus, da es, wie in Oerel wiederum gezeigt wurde, bei zwei bewaldeten Frühweichsel-Interstadialen bleiben muß. Die teilweise sehr hohen Werte von Alnus (bis $62 \%$ im Bruchwald) und Picea (bis $47 \%$ ) 
im Amersfoorter „Brörup” schließen dessen Synchronisation mit dem Odderade wohl aus, so daß die ZAGWIJNsche Ansprache als Brörup richtig sein muß. Ebenso muß man die Korrelierung des „Amersfoort" mit dem sehr schwachen und unbewaldeten dänischen Roedebaek-Interstadial (ANDERSEN 1961) verneinen, denn das „Amersfoort" war nicht nur voll bewaldet, sondern weist in Bohrung 2 sogar bis $\mathrm{zu} 8 \%$ Eichenmischwaldpollen auf, und zwar im Übergangstorf, so daß er nicht umgelagert worden sein kann, sondern aus der Umgebung eingeweht sein muß.

Ein solcher Klimaunterschied zwischen den Niederlanden und Jütland ist kaum vorstellbar, vor allem fehlen auch aus dem dazwischengelegenen nordwestdeutschen Raum bislang jegliche Hinweise auf ein Interstadial zwischen Eem und Brörup, obwohl es inzwischen eine größere Zahl von Sequenzen EemBrörup gibt (z. T. unveröffentlicht). Es bleibt nur die Konsequenz, die niederländischen „Amersfoort” und "Brörup" zusammen dem Brörup im dänischen (und deutschen) Sinne gleichzusetzen, wie es bereits verschiedene Autoren (z. B. GRÜGER 1979b, MENKE 1982) angenommen haben. In den meisten Pollendiagrammen des Brörup im dänischen Sinne läßt sich anhand der Vegetation ein Klimarückschlag (WF II a $a_{2}$ ) erkennen, in Oerel ist er allerdings relativ schwach. Dieser Rückschlag führt bei uns jedoch nicht zur Entwaldung. Die in Amersfoort (vgl. ZAGWIJN 1961, Bohrung 3) zwischen „Amersfoort” und „Brörup” liegende Sandschicht dürfte diesem Rückschlag entsprechen. Ihre Ausprägung muß jedoch besondere lokale Ursachen haben, denn auch dort läuft die Bewaldung offenbar ohne Unterbrechung weiter; Initialphasen einer oberhalb der Sandschicht neu beginnenden Bewaldung lassen sich aus dem Pollendiagramm nicht ablesen, so daß der scharf begrenzte Sand eine brörupzeitliche Einschwemmung sein muß.

Gestützt wird die hier dargelegte Gliederung des Weichsel-Frühglazials mit nur zwei großen Interstadialen durch die in den letzten Jahren bearbeiteten lang durchlaufenden Spätpleistozänprofile von Grande Pile (Woillard 1975), Les Echets (DE BEAUlieu \& REILLE 1984), Sulzberg (WELTEN 1981) und Samerberg (GRÜGER 1979b) im südlichen Mitteleuropa, wo diese beiden großen Interstadiale teilweise bereits den Charakter von Interglazialen annehmen; eine vergleichbare Abfolge zeigen auch die Temperaturkurven aus Tiefseekernen (SHACKLETON 1977).

Die Parallelen $\mathrm{zu}$ den unbewaldeten Interstadialen Oerel und Glinde sind bislang unsicher. Möglich ist eine Korrelierung des Oerel mit dem Ognon und damit wohl auch mit dem Dürnten und dem 3. Interstadial am Samerberg (vgl. S. 31). Will man die Interstadiale Oerel und Glinde im Standardprofil Grande
Pile wiederfinden, so kommt nur die Synchronisierungs-Alternative 1 von WOILLARD 1979, S. 63, in Frage, und Brörup und Odderade wären dann mit St. Germain I und II gleichzusetzen, wofür auch fast alle anderen Befunde in Europa sprechen.

Den Schlüssel für die Abfolge der Weichsel-Interstadiale müßte das Profil Königsaue (MANIA \& TOEPFER 1973) liefern, sobald von dort eine pollenanalytische Bearbeitung vorliegt, die eine sichere Korrelation ermöglicht. Die bisherigen Untersuchungen dort haben 2 große Frühweichsel-Interstadiale ergeben, auf die 5 schwächere Interstadiale folgen, welche Oerel und Glinde sowie Moershoofd, Hengelo und Denekamp entsprechen könnten. Darüber liegt die spätglaziale Folge. Bisher spricht nichts gegen die Vollständigkeit der Serie von Königsaue, und mit den neu hinzukommenden Interstadialen Oerel und Glinde ist die in Königsaue nachgewiesene Zahl der Interstadiale auch außerhalb dieser Lokalität exakt erreicht. Die daraus folgende Korrelation zwischen Oerel und Königsaue ist in Abb. 12 dargestellt.

Im klimatischen Vergleich von Oerel und Glinde ist besonders das Auftreten der etwas wärmebedürftigen Filipendula interessant, die im Oerel zeitweise eine geschlossene Kurve erreicht, im Glinde dagegen nur mit einem einzigen Pollenkorn nachgewiesen ist. In die gleiche Richtung weist auch das ähnliche Verhalten von Juniperus. Daraus darf man wohl auf eine im Glinde niedrigere Sommertemperatur schließen.

Die Grenzziehung zwischen Weichsel-Frühglazial und Weichsel-Pleniglazial erfolgt im Profil Oerel (abweichend von MENKE \& TYNNI 1984) zwischen Odderade-Interstadial und Schalkholz-Stadial. Nachdem die SEQS am Standardprofil Samerberg einen entsprechenden Vorschlag für die Grenze Unteres Würm/Mittleres Würm gemacht hat (CHALINE \& JERZ 1984), soll diesem gefolgt werden, zumal das Pleniglazial besser mit einem Stadial als mit einem Interstadial beginnt und diese Grenzziehung bereits von einigen Autoren verwendet worden ist.

Der Begriff „Interstadial” ist im vorangehenden so benutzt worden, daß er eine deutliche relative Klimaverbesserung kennzeichnet. Der klimatische und vegetationsgeschichtliche Inhalt eines bestimmten Interstadials kann je nach geographischer Breite sehr unterschiedlich sein und etwa im Brörup von $\mathrm{S}$ nach $\mathrm{N}$ von Eichenmischwäldern über boreale Nadelwälder bis zur Waldlosigkeit reichen. Entsprechend fließend ist auch die Definition Interstadial-Interglazial; sie ist deshalb nur regional zu fassen. In einem bestimmten Gebiet, wie etwa Norddeutschland, gab es andererseits im Verlauf einer Kaltzeit sehr unterschiedliche Klima- und Vegetationsverhältnisse. Deutliche relative Klimaverbesserungen, die als Interstadial 


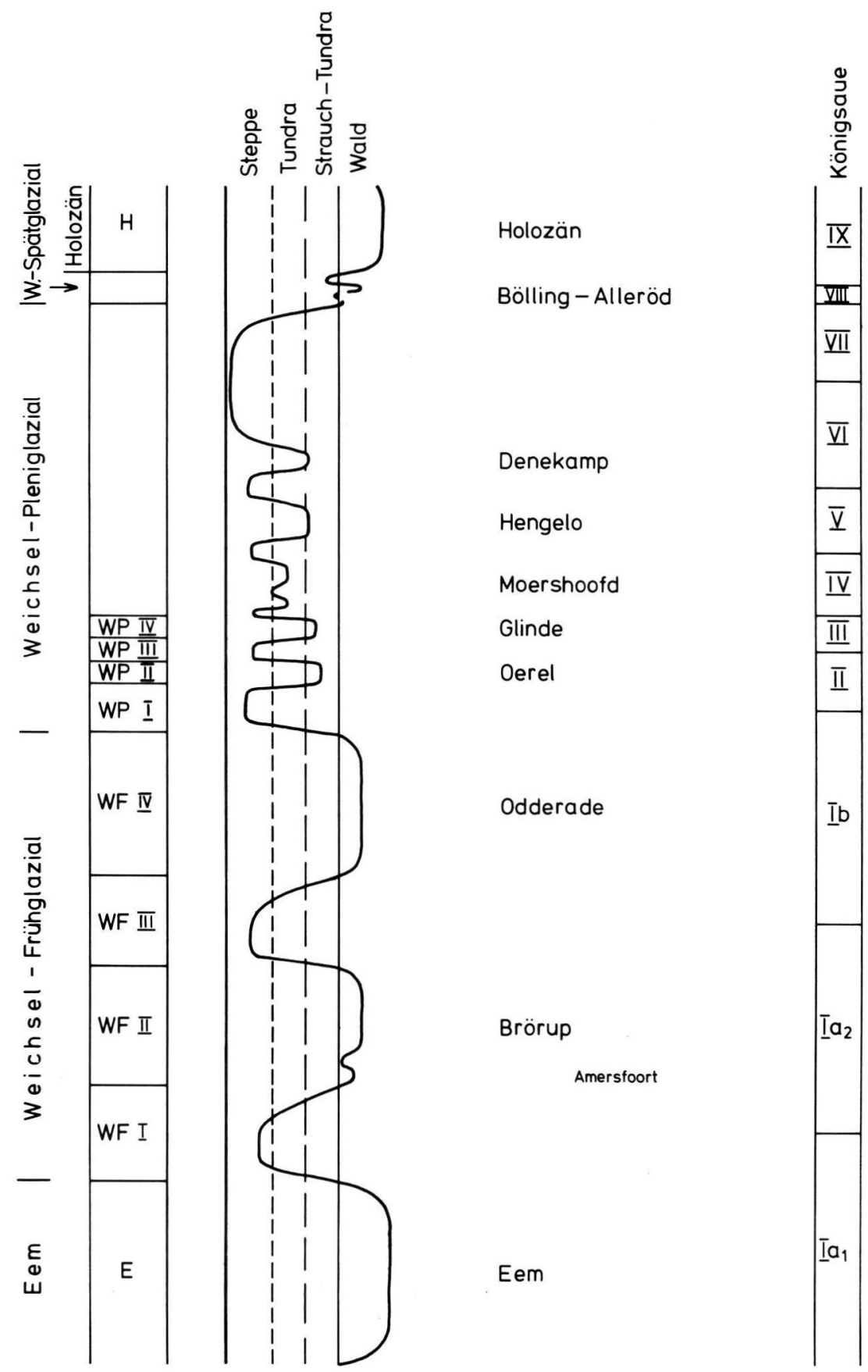

Abb. 12: Schematische Darstellung der Weichsel-Interstadiale und ihres Vegetationscharakters im norddeutschen Tiefland. Rechts zum Vergleich die vermutete Korrelation mit dem Profil Königsaue im östlichen Harzvorland.

bezeichnet werden, können von einer Polarsteppe bis zur geschlossenen (waldfreien) Vegetationsdecke oder von dieser zur Strauchtundra oder zum Wald führen. Das bedeutet, daß nicht nur Klima und Vegetation der verschiedenen Interstadiale sehr differieren, sondern auch, daß im gleichen Gebiet die stadiale Vegetation in einem Zeitbereich die gleiche sein kann wie die interstadiale Vegetation zu anderer Zeit; z. B. ist im niedersächsischen Tiefland die Strauchtundra im Oerel- oder Glinde-Interstadial ähnlich der im 
Stadial der Jüngeren Dryas-Zeit. Ein Teil der als Moershoofd-Interstadial eingestuften Vorkommen dürfte klimatisch (nicht chronologisch) etwa den Herning-und Rederstall-Stadialen entsprechen. Entscheidend für die Anwendung des Begriffs Interstadial ist eine möglichst scharfe Abhebung von vorangehenden und folgenden Abschnitten. In Oerel kommt zur Änderung der Vegetation noch der Wechsel im Sediment von Sand bzw. Mudde zum Torf, so daß selbst für die beiden waldfreien oberen Interstadiale die Abgrenzung sehr klar ist. Das muß aber nicht immer so sein, denn z. B. in den niederländischen jüngeren pleniglazialen Interstadialen wurde nur selten richtiger Torf gebildet.

Wie gezeigt wurde, konnten in der Hohlform von Oerel neue Interstadiale aus der bislang dunklen Zeit des frühen Weichsel-Pleniglazials beschrieben werden. Weitere lang durchlaufende Profile aus dem nördlichen Mitteleuropa sind erforderlich, um sicherzustellen, ob damit die Zahl der Weichsel-Interstadiale vollständig ist, wie die Korrelation mit Königsaue vermuten läßt. In $\mathrm{Abb} .12$ sind unsere augenblicklichen Vorstellungen über die Klimaentwicklung in der Weichsel-Kaltzeit dargestellt. Während sich das Bild von der interstadialen Vegetation vor allem durch pollenanalytische Untersuchungen immer weiter verdichtet, herrscht über das Klima der meisten Stadiale jedoch nach wie vor große Unsicherheit.

\section{Schriftenverzeichnis}

AARIO, L. (1940): Waldgrenzen und subrezente Pollenspektren in Petsamo Lappland. - Ann. Acad. Sci. Fenn. A 54 (8): 1-120; Helsinki.

ANDERSEN, S. Th. (1961): Vegetation and its Environment in Denmark in the Early Weichselian Glacial (Last Glacial). - Danmarks Geologiske Unders $\varnothing$ gelse, R. II 75: 1-175; Kopenhagen.

AVERDIECK, F.-R. (1967): Die Vegetationsentwicklung des Eem-Interglazials und der Frühwürm-Interstadiale von Odderade/Schleswig-Holstein. - Fundamenta B, 2; Frühe Menschheit und Umwelt 2: 101-125; Köln.

de Beaulieu, J.-L. \& Reille, M. (1984): A long Upper Pleistocene pollen record from Les Echets, near Lyon, France. - Boreas, 13: 111-132; Oslo.

BEHRE, K.-E. (1962): Pollen- und diatomeenanalytische Untersuchungen an letztinterglazialen Kieselgurlagern der Lüneburger Heide. - Flora, 152: 325-370; Jena.

- (1966): Untersuchungen zur spätglazialen und frühpostglazialen Vegetationsgeschichte Ostfrieslands. Eiszeitalter und Gegenwart, 17: 69-84; Öhringen.

- (1970): Die Flora des Helgoländer Süßwasser-,,Töcks”, eines Eem-Interglazials unter der Nordsee. - Flora, 159: 133-146; Jena.
BEHRE, K.-E. (1974): Die Vegetation im Spätpleistozän von Osterwanna/Niedersachsen (Pollen- und Makrorestuntersuchungen aus Eem- und Frühweichsel). - Geol. Jb.; A 18: 3-48; Hannover.

BeNDA, L. \& SCHNEEKLOTH, H. (1965): Das Eem-Interglazial von Köhlen, Krs. Wesermünde. - Geol. Jb., 83: 699-716; Hannover.

Chaline, J. \& Jerz, H. (1984): Stratotypen des' WürmGlazials. - Eiszeitalter und Gegenwart, 35: 185-206; Hannover.

EHLERS, J. (1979): Gefügekundliche und sedimentpetrographische Untersuchungen im Pleistozän der Kreidegrube Hemmoor (Nordwest-Niedersachsen). - Geol. Jb., A 49: 27-37; Hannover.

ERD, K. (1973): Pollenanalytische Gliederung des Pleistozäns der Deutschen Demokratischen Republik. - Z . geol. Wiss. 1: 1087-1103; Berlin (Ost).

Frenzel, B. (1980): Das Klima der letzten Eiszeit in Europa. - In: Oeschger, H., Messerli, B. \& Svilar, M. (Hg.): Das Klima: 45-63; Berlin, Heidelberg, New York (Springer).

GRÜGER, E. (1979a): Spätriß, Riß/Würm und Frühwürm am Samerberg in Oberbayern - ein vegetationsgeschichtlicher Beitrag zur Gliederung des Jungpleistozäns. - Geologica Bavarica, 80: 5-64; München.

- (1979b): Die Seeablagerungen vom Samerberg/Obb. und ihre Stellung im Jungpleistozän. - Eiszeitalter und Gegenwart, 29: 23-34; Hannover.

HAGEDORN, H. \& LADE, U. (1981): Lithostratigraphie, Genese und Basisrelief jungpleistozäner Sedimente in Nordwestdeutschland. - Abschlußbericht zum gleichnamigen DFG-Projekt 1979-1981, 35 S. - Archiv Geogr. Institut Univ. Würzburg. - [Unveröff.].

VAN DER HAMMEN, T. (1971): The Upper Quaternary stratigraphy of the Dinkel valley. - Mededelingen Rijks Geolog. Dienst NS; 22: 59-72; Maastricht.

-, Maarleveld, G. C., Vogel, J. C. \& Zagwijn, W. H. (1967): Stratigraphy, Climatic Succession and Radiocarbon Dating of the Last Glacial in the Netherlands. Geologie en Mijnbouw, 46: 79-95; s'Gravenhage.

HÖFLE, H.-CHR. (1982): Erläuterungen zu Blatt 2624 Hollenstedt. Geologische Karte von Niedersachsen 1:25000, 75 S.; Hannover (Nieders. L.-Amt Bodenforsch.).

- \& LADE, U. (1983): The stratigraphic position of the Lamstedter Moraine within the Younger Drenthe substage (Middle Saalian). - In: EHLERS, J. (Hg.): Glacial Deposits in North-West Europe: 343-346; Rotterdam (Balkema).

— \& SCHLENKER, B. (1979): Das Pleistozänprofil der Kreidegrube Hemmoor bei Stade (Elbe-Weser-Dreieck). - Geol. Jb., A 49: 3-25; Hannover.

KolstruP, E. \& WijMSTRA, T. A. (1977): A palynological investigation of the Moershoofd, Hengelo and Denekamp interstadials in the Netherlands. - Geologie en Mijnbouw 56: 85-102; s' Gravenhage. 
KuSTER, H. \& MEYeR, K.-D. (1979): Glaziäre Rinnen im mittleren und nordöstlichen Niedersachsen. - Eiszeitalter und Gegenwart, 29: 135-156; Hannover.

LADE, U. (1980): Quartärmorphologische und -geologische Untersuchungen in der Bremervörder - Wesermünder Geest. - Würzburger Geograph. Arb., 50: 176 S.; Würzburg.

- (1985): Die quartären Deckschichten zwischen Cuxhaven und Bremerhaven (Hohe Lieth). - In: SimON, P. et al.: Aufsuchung und Untersuchung von Schwermineralanreicherungen im Gebiet zwischen Cuxhaven und Bremerhaven (Hohe Lieth). - Ber. Arch. Nieders. L. -Amt Bodenforsch. Hannover. - [Unveröff.].

- \& Hagedorn, H. (1982): Sedimente und Relief einer eiszeitlichen Hohlform bei Krempel (Elbe-Weser-Dreieck). - Eiszeitalter und Gegenwart, 32: 93-108; Hannover.

LAMB, H. F. (1984): Modern pollen spectra from Labrador and their use in reconstructing holocene vegetational history. - J. Ecology, 72: 37-59; Oxford.

LiCHTI-Federovich, S. \& Ritchie, J. C. (1968): Recent pollen assemblages from the western interior of Canada. Review of Palaeobotany Palynology, 7: 297-344; Amsterdam.

LIEDTKE, H. (1981): Die nordischen Vereisungen in Mitteleuropa; 2. Aufl. - Forsch. Dt. Landeskunde, 204, 307 S.; Trier.

MAniA, D. \& Toepfer, V. (1973): Königsaue. - 164 S.; Berlin (VEB Deutscher Verlag d. Wiss.).

VAN DER MeER, J., Slotboom, R. T. \& DE ViRes-BRUYNSTEen, J. M. E. (1974): Lithology and palynology of Weichselian alluvial fan deposits near Eerbeek, The Netherlands. - Boreas, 13: 393-402; Oslo.

MenKe, B. (1976): Neue Ergebnisse zur Stratigraphie und Landschaftsentwicklung im Jungpleistozän Westholsteins. - Eiszeitalter und Gegenwart, 27: 53-68; Öhringen.

- (1980): Vegetationskundlich-ökologisches Modell eines Interglazial-Glazial-Zyklus in Nordwestdeutschland. Phytocoenologia, 7: 100-120; Stuttgart.

- (1982): On the Eemian interglacial and the Weichselian glacial in Northwestern Germany (Vegetation, Stratigraphy Palaeosols, Sediments). - Quaternary Studies in Poland, 3: 61-68; Warszawa; Poznań.

- \& Trnni, R. (1984): Das Eeminterglazial und das Weichselfrühglazial von Rederstall/Dithmarschen und ihre Bedeutung für die mitteleuropäische Jungpleistozän-Gliederung. - Geol. Jb., A 76: 120 S.; Hannover.

Meusel, H., Jäger, E., Rauschert, St. \& Weinert, E. (1978): Vergleichende Chronologie der zentraleuropäischen Flora, 2 (Text- u. Tafelband); 418 S.; Jena (Fischer)
MEYER, K.-D. (1982): Ėrläuterungen zu Blatt 2524 Buxtehude. - Geol. Kt. Niedersachsen 1:25000, 120 S.; Hannover (Nieders. L.-Amt Bodenforsch.).

- \& Schneekloth, H. (1973): Geol. Kt. Niedersachsen $1: 25000$, Blatt Neuenwalde Nr. 2318; mit Erläuterungen, Hannover (Nieders. L.-Amt Bodenforsch.).

Peschke, P. (1983): Pollenanalysen der Schieferkohlen von Herrnhausen (Wolfratshausener Becken/Obb.) - ein Beitrag zum Problem interglazialer Ablagerungen in Oberbayern. - Geologica Bavarica, 84: 107-121; München.

PICARD, K. (1959): Gliederung pleistozäner Ablagerungen mit fossilen Böden bei Husum/Nordsee. - Neues Jahrb. Geol., Paläont., Monatsh.: 259-272; Stuttgart.

Punt, W. \& Den Breejen, P. (1981): Linaceae. - In: Punt, W. \& Clarke, G. C. S. (Eds.): The Northwest European Pollen Flora, 3: 75-116; Amsterdam, Oxford, New York.

SCHNEEKLOTH, H. (1966): Ergebnisse weiterer Untersuchungen an den interstadialen Ablagerungen der WeichselEiszeit in Oerel/Krs. Bremervörde. - Z. dt. geol. Ges., 116: 773-796; Hannover.

SELLE, W. (1962): Geologische und vegetationskundliche Untersuchungen an einigen wichtigen Vorkommen des letzten Interglazials in Nordwestdeutschland. - Geol. Jb., 79: 295-352; Hannover.

- \& Schneekloth, H. (1965): Ergebnisse einer Kernbohrung in Oerel, Krs. Bremervörde; drei Interstadiale über Ablagerungen des Eem-Interglazials. - Z $\mathrm{Z}$ dt. geol. Ges., 115: 109-117; Hannover.

Shackleton, N. J. (1977): The oxygen isotope stratigraphic record of the Late Pleistocene. - Phil. Trans. R. Soc. London B 280: 169-182; London.

SINDOWSKI, K.-H. (1969): Erl. Bl. 2117 Altenwalde. Geol. Kt. Niedersachsen 1:25000: 63 S.; Hannover (Nieders. L.-Amt Bodenforsch.).

Teunissen, D. \& Teunissen-van Oorschot, H. (1974): Eine interstadiale Torfschicht bei Nijmegen (Niederlande) und deren Bedeutung für die Erklärung der dortigen Landschaftsmorphologie. - Geologie en Mijnbouw, 53: 393-400; s'Gravenhage.

Welten, M. (1981): Veränderung und Vernichtung der anspruchsvollen Gehölze am Beginn der letzten Eiszeit und die Korrelation der Frühwürm-Interstadiale in Mittel- und Nordeuropa. - Eiszeitalter und Gegenwart, 31: 182-202; Hannover.

- (1982): Pollenanalytische Untersuchungen im Jüngeren Quartär des nördlichen Alpenvorlandes der Schweiz. - Beitr. z. Geolog. Karte der Schweiz NF, 156: 174 S.; Bern.

Wijmstra, T. A. (1969): Palynology of the first 30 metres of a $120 \mathrm{~m}$ deep section in northern Greece. - Acta botan. Neerlandica, 18: 511-527; Amsterdam. 
WoIllard, G. (1975): Recherches palynologiques sur le pleistocene dans l'est de la Belgique et dans les Vosges Lorraines. - Acta Geographica Lovaniensia, 14: 118 S.; Louvain.

- (1978): Grande Pile Peat Bog: A continuous Pollen Record for the last 14000 Years. - Quaternary Research, 9: 1-21; Washington.

- (1979): The last interglacial-glacial cycle at Grande Pile in Northeastern France. - Bulletin Soc. belge de Géologie, 88: 51—69; Brüssel.

WoldSTEDT, P. (1950): Norddeutschland und angrenzende Gebiete im Eiszeitalter. - 464 S.; Stuttgart (Köhler).
ZAGWIJN, W. H. (1961): Vegetation, climate and radiocarbon datings in the Late Pleistocene of the Netherlands, Part I: Eemian and Early Weichselian. - Mededelingen Geol. Stichting, N. S. 14: 15—45; Maastricht.

- (1974): Vegetation, climate and radiocarbon datings in the Late Pleistocene of the Netherlands, Part II: Middle Weichselian. - Mededel. Rijks Geolog. Dienst, NS 25: 101-111; Maastricht.

Manuskript eingegangen am 23.5.85. 